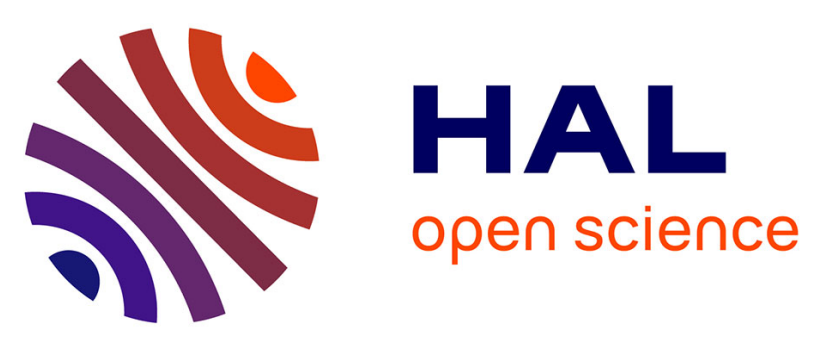

\title{
Fracture and debonding of a thin film on a stiff substrate: analytical and numerical solutions of a 1d variational model
}

Andrés Alessandro León Baldelli, Blaise Bourdin, Jean-Jacques Marigo, Corrado Maurini

\section{To cite this version:}

Andrés Alessandro León Baldelli, Blaise Bourdin, Jean-Jacques Marigo, Corrado Maurini. Fracture and debonding of a thin film on a stiff substrate: analytical and numerical solutions of a $1 \mathrm{~d}$ variational model. 2011, pp.on line. hal-00647863

\section{HAL Id: hal-00647863 \\ https://hal.science/hal-00647863}

Submitted on 2 Dec 2011

HAL is a multi-disciplinary open access archive for the deposit and dissemination of scientific research documents, whether they are published or not. The documents may come from teaching and research institutions in France or abroad, or from public or private research centers.
L'archive ouverte pluridisciplinaire HAL, est destinée au dépôt et à la diffusion de documents scientifiques de niveau recherche, publiés ou non, émanant des établissements d'enseignement et de recherche français ou étrangers, des laboratoires publics ou privés. 


\title{
A.A. Leon Baldelli · B. Bourdin · J.-J. Marigo · C. Maurini \\ Fracture and debonding of a thin film on a stiff substrate: analytical and numerical solutions of a 1d variational model
}

Version: November 15, 2011 / Received: date / Accepted: date

\begin{abstract}
We study multifissuration and debonding phenomena of a thin film bonded to a stiff substrate using the variational approach to fracture mechanics. We consider a reduced one-dimensional membrane model where the loading is introduced through uniform inelastic (e.g. thermal) strains in the film or imposed displacements of the substrate. Fracture phenomena are accounted for by adopting a Griffith model for debonding and transverse fracture. On the basis of energy minimization arguments, we recover the key qualitative properties of the experimental evidences, like the periodicity of transverse cracks and the peripheral debonding of each regular segment. Phase diagrams relate the maximum number of transverse cracks that may be created before debonding takes place as a function of the material properties and the sample's geometry. The theoretical results are illustrated with numerical simulations obtained through a finite element discretization and a regularized variational formulation of the Ambrosio-Tortorelli type, which is suited to further extensions in two-dimensional settings.
\end{abstract}

Keywords Variational approach $\cdot$ Thin films $\cdot$ Fracture $\cdot$ Delamination $\cdot$ Energy minimization

\section{Introduction}

Thin film systems are widely present in today's engineering solutions. Coatings, surface treatments, thermal barriers and paintings are just to mention some. These systems are often characterized by the presence of a substrate, whose duty is to support the structural loading, and one or several layers, somehow bonded to the substrate, meant to impart a particular superficial behavior to the final assembly. The layer, or "film", as it will be called henceforth, and the substrate are often of very different material and geometric characteristics. Usually, the film is much thinner than the substrate and its stiffness and thermal expansion coefficients may differ from those of the substrate by several orders of magnitude. Because of the assembly procedures of the final system and multi-physical loadings under working conditions (e.g. thermal loads or humidity), this mismatch may lead to significant stresses in the film and eventually lead to material failure, which can be manifested by a plethora of different and complex modes of cracking.

A.A. Leon Baldelli · C. Maurini

UPMC Univ Paris 6, UMR 7190, Institut Jean Le Rond d'Alembert, Boite courrier 161-2, 4 Place Jussieu, F-75005, Paris, France CNRS, UMR 7190, Institut Jean Le Rond d'Alembert, Boite courrier 161-2, 4 Place Jussieu, F-75005, Paris, France

E-mail: leon@dalembert.upmc.fr, corrado.maurini@upmc.fr

B. Bourdin

Department of mathematics and Center for Computation \& Technology, Louisiana State University, Baton Rouge, LA 70803, USA.

E-mail: bourdin@1su.edu

J.-J. Marigo

Ecole Polytechnique, Laboratoire de Mécanique des Solides, 91128, Palaiseau, France

E-mail: marigo@lms.polytechnique.fr 
A detailed review of possible failure modes has been given in [1]. Isolated cracks or network of channeling fractures may develop and propagate in the overlying film, cracks originating from within the film may penetrate into the substrate, interfacial cracks may develop in the bonding layer. Specifically, multifissuration and debonding are observed in a multitude of physical systems such as rocky materials and asphalts [2], microelectromechanical systems, or through drying of colloidal suspensions [3]. Considering that these systems possess very different constitutive laws and characteristics lengths spanning multiple orders of magnitude, one can argue that multifissuration and debonding are not specific to a particular material microstructure and should be amenable to a macroscopic description.

A common observation is that, under uniaxial loading of the specimen, a periodic network of cracks appears [4] and debonding of the film eventually takes place starting from the edges. With this starting assumption, numerous studies have been performed either with energy minimization criterions or maximum-stress criterions. Multifissuration of thin films has been studied in [5], without accounting for the non-linear interaction with the substrate that provokes delamination. In that work identical mechanical properties for the film and substrate have been considered. Crack spacing for a pavement layer has been studied in [2] using an elasto-plastic-cohesive-frictional model for the interface. In [6] a critical stress threshold activates debonding, then an energy minimization argument is used to solve the segmentation problem. A comparison of different constitutive laws that may activate debonding are studied in [7]. In all those examples, residual friction remains where the film is debonded. The priori knowledge of the debonded area's topology and of the crack spacing is also central to the analysis also in [8] and [9].

A proper description of the complex cracking phenomena observed in thin films demands to correctly deal with complex crack geometries, crack nucleation and evolution, which eventually may turn out to be non-smooth in space-time. These issues are known to be fundamental difficulties for the classical approach to fracture, which is based on the analysis of the propagation of a single preexisting crack along a simple, and usually pre-defined path. As a result, although thin film systems are of a great scientific and industrial interest, a general and rigorous modeling framework able to provide accurate tools for their theoretical understanding and predictive numerical simulation is still missing. A key reference for the linear fracture mechanics of thinfilm systems is the work of Xia and Hutchinson [10], where the authors use a reduced two-dimensional membrane model for the film and the substrate stiffness is accounted for as an equivalent elastic foundation. In this setting, the dimensionless parameters of the model are calibrated through finite element computations. The propagation of a single straight crack, that of an array of cracks (with the typical stop-and-go phenomenon), curved and spiral cracks were found as particular solutions of the equation of the critical energy release rate criterion, namely $G=G_{c}$.

The aim of our work is to study the problem of transverse cracking and debonding of a thin film elastically bonded on a stiff substrate. To overcome the limits of classical linear fracture mechanics regarding crack nucleation and its evolution in space-time, we adopt a variational point of view. We consider a reduced model similar to that of [10], revisiting it in the framework of the variational approach to fracture mechanics as stated in [11, 12, 13]. We postulate that the cracked state of the system is given by a unilateral global minimization principle of a total energy functional consisting of the sum of a bulk contribution, due to the elasticity of the film and the bonding layer, and surface terms associated to the energy required to create a transverse or debonding cracks, according to Griffith model. We focus here on a $1 D$ study, which includes both analytical and numerical results. Many of the assumptions that are often postulated, namely the equidistribution of cracks, the topology of the debonded region, its activation from the boundaries, are obtained as outcomings of the variational analysis. Details about the organization of the paper and its main results are given in the following Section.

The present paper shares the key ideas of the stream of works on the variational analysis of non-linear continua, to which Professor Del Piero gave several fundamental contributions [14,15],16, 17]. Our work is heartily dedicated to him in occasion of his retirement.

\section{Organization of the paper and main results}

We start fixing some basic notation. We follow the convention of the journal to denote scalars, vectors and tensors quantities (and analogously fields) with italic serif, bold serif and sans serif fonts like $u, \mathbf{x}$ and e, respectively. The derivative of a function of one variable will be indicated by a prime sign and the Gateaux derivative of a functional $E(u, \ldots)$ in the direction $v$ will be noted $D_{u} E(u, \ldots)(v)$. To keep the notation as light 
$2 D$

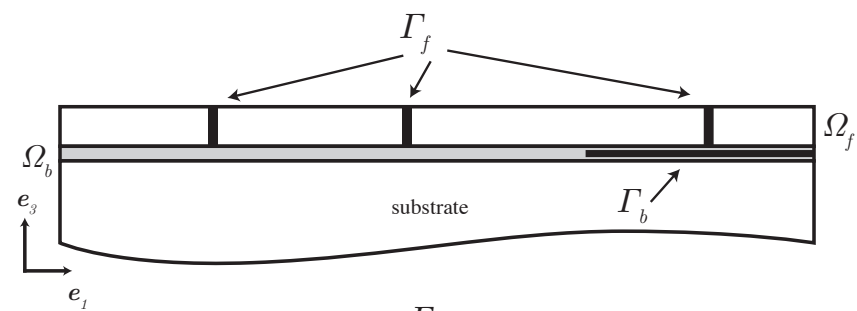

$1 D$

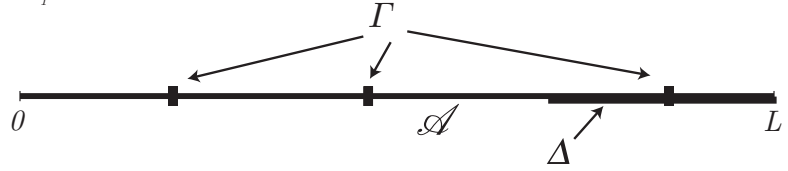

Fig. $12 D$ : A cross-section of the $3 D$ system: the film $\Omega_{f}$ is bonded to a stiff substrate by the means of the bonding layer $\Omega_{b}$. Transverse cracks $\Gamma_{f}$ debonding cracks $\Gamma_{b}$ may develop in the film and in the bonding layer. $1 D$ reduction: in the reduced uniaxial elastic bar of axis $\mathscr{A}$, transverse and debonding cracks are identified with $\Gamma$ and $\Delta$.

as possibl, with an abouse of notation, we will denote sometimes different functions with the same symbol, provided that the number of arguments is different. Finally, we denote \# $(\cdot)$ and $\mathscr{L}(\cdot)$ the cardinal number (0-dimensional measure in $\mathbb{R}^{N}$ ) and the length (1-dimensional measure in $\mathbb{R}^{N}$ ) of a set.

We consider the system sketched in Figure 1, where a film is bonded on a stiff substrate through a thin bonding layer. We study the appearance of transverse cracks $\Gamma$ across the film cross-section and interfacial cracks $\Delta$ along the bonding layer. Denoting by the scalar field $u$ the membrane displacement of the film occupying the $1 D$ domain $\mathscr{A}$, we adopt a one-dimensional reduced model, which assumes that the total energy of the system is given by:

$$
\mathscr{E}_{t}(u, \Gamma, \Delta):=\int_{\mathscr{A} \backslash \Gamma} \frac{1}{2} K\left(u^{\prime}(x)-\bar{\varepsilon}_{t}\right)^{2} d x+\int_{\mathscr{A} \backslash \Delta} \frac{1}{2} k\left(u-\bar{u}_{t}\right)^{2} d x+G_{\Gamma} \#(\Gamma)+G_{\Delta} \mathscr{L}(\Delta),
$$

where $K$ is its membrane stiffness, $k$ is an equivalent stiffness of the bonding layer, $G_{\Gamma}$ is the energy required to create a transverse crack (transverse fracture toughness), $G_{\Delta}$ is the energy required to debond a unit length interval (debonding toughness), \# $(\Gamma)$ is the number of transverse cracks, and $\mathscr{L}(\Delta)$ is the total length of the debonded regions. As common in thin-film systems, the loading is represented by inelastic strains $\bar{\varepsilon}_{t}$ in the film (e.g. thermally induced deformations) or an imposed displacement of the substrate $\bar{u}_{t}$. We consider only the case in which $\bar{\varepsilon}_{t}$ is uniform and $\bar{u}_{t}$ varies at most linearly throughout the domain.

The model is briefly presented in Section 3. After a dimensional analysis, we show that the energy (1) depends on two dimensionless parameters representing the geometric and the material properties, that we choose as a scaled dimensionless film length $L$ and a relative fracture toughness $\gamma$, and a single parameter for the loading, which we take as a scaled inelastic strain $\bar{\varepsilon}_{t}$. A variational formulation of the quasi-static evolution problem for $(u, \Gamma, \Delta)$ is given at the end of Section 3 It consists in requiring the conditions of unilateral global stability, irreversibility, and energy balance, as usually postulated in the variational theories of rate-independent processes [18].

Section 4 focusses on the static version of the variational problem, $i$. $e$. on looking for the energy minimizers at a fixed loading, without reference to any previous history or irreversibility condition. The solutions of the quasi-static problem are illustrated in Section 5. They are obtained by applying the further selection criteria of irreversibility and energy balance. We resume below the main qualitative results that are presented in the analytical part of the work:

- The solution of the problem without debonding (i.e. for infinite debonding toughness) is in the form of an equally spaced array of transverse cracks partitioning the film in $n$ segments of length $L_{i}=L / n$. We give analytically the displacement field in each segment as well as the number of transverse cracks as a function of the loading (Proposition 1). In a quasi-static evolution, because of the irreversibility condition, there is a cascade of fracture phenomena in which each segment splits in two parts by a transverse fracture in its middle. Hence, the number of segments are in the form $n=2^{j}, j \in \mathbb{N}$ (Section 5.1).

- In a film with finite debonding toughness divided in $n$ regular segments by $n-1$ transverse fractures, debonding simultaneously takes place at (one or both) the ends of each segment. The critical loading 
level at which this occurs is given analytically. The length of the single bonded interval of each segment is univocally determined as a function of the loading. However, solutions obtained by transferring the debonded regions from one end of a segment to any other end of any other segment are energetically equivalent (Propositions 2 and 3 ).

- We illustrate the possible cracking scenarios with coupled transverse fractures and debonding for specific values of the geometric and material parameters $L$ and $\gamma$. For the static problem, we show that for low values of the load the global minimizer of the total energy is a solution without debonding, while for high values of the load debonding without transverse fracture is energetically preferred. Solutions with coupled transverse fractures and debonding are energy minimizers for intermediate loadings (see Proposition 4 and Figure 7). In a quasi-static evolution, there is cascade of $k$ transverse fracture phenomena after which the film is split in $n=2^{k}$ segments, followed by the debonding. After that debonded starts, no further transverse fracture appears. A numerically evaluated phase diagram (Figure 13) gives the number $k$ of transverse fracture phenomena before debonding as a function of $L$ and $\gamma$.

Section 6 focuses on the numerical solution of the quasi-static problem. The numerical implementation is based on a regularized formulation of the variational problem and a finite element discretization, which extends to the case of the thin film energy (1) the approach of [19,20]. The quasi-static evolutions are numerically obtained by replacing the unilateral global stability principle, with a weaker unilateral local stability condition. On one hand, this is done because global minimization of non convex functionals in highdimensional vector spaces is numerically out of reach. On the other hand, the corresponding solutions to the evolution regularized model may be mechanically interpreted as those of a non-local damage model, where the regularization parameter can be regarded as an internal length that influences the critical loads at which fractures appear. The comparison of the solution of the quasi-static evolution problem obtained analytically and numerically allows us to validate the numerical approach and identify some shortcomings.

\section{Formulation of the problem}

3.1 The three-dimensional system: geometry, materials and loadings.

We consider a thin film elastically bonded to the upper surface $\omega \subset \mathbb{R}^{2}$ of a stiff substrate. Referring to the sketch in Figure 1 , we denote by $\Omega_{f}=\mathscr{A} \times(-w / 2, w / 2) \times\left(0, h_{f}\right) \subset \mathbb{R}^{3}$ and $\Omega_{b}=\mathscr{A} \times(-w / 2, w / 2) \times$ $\left(0,-h_{b}\right) \subset \mathbb{R}^{3}$ the domains occupied by the film and the bonding layer in their reference configurations, $\mathscr{A}=[0, L]$ being the central axis of the film/bonding layer interface. We suppose that both the film and the bonding layer are isotropic linear elastic brittle materials, whose Young modulus, Poisson ratio and fracture toughness are denoted by $\left(E_{f}, v_{f}, G_{f}\right)$ and $\left(E_{b}, v_{b}, G_{b}\right)$. We distinguish two families of possible cracks: (i) debonding cracks $\Gamma_{b} \subseteq \Omega_{b}$ in the bonding layer and (ii) transverse cracks $\Gamma_{f} \subset \Omega_{f}$ inside the film. We suppose that transverse cracks are in the form $\Gamma_{f} \equiv(\Gamma \subset \mathscr{A}) \times(-w / 2, w / 2) \times\left[0, h_{f}\right]$, i.e. that they completely cut a transversal cross-section of the film perpendicularly to the film/bonding layer interface. Similarly, we assume that the debonding cracks develop throughout the width of the strip being in the form $\Gamma_{b} \equiv(\Delta \subset \mathscr{A}) \times$ $(-w / 2, w / 2) \times\left(z \in\left(-h_{b}, 0\right)\right)$. In the following we identify them by the parts of the domain $\mathscr{A}$ that are fractured $(\Gamma)$ or delaminated $(\Delta)$.

We consider two types of loadings. The first one models the effect of the substrate through an imposed displacement $\overline{\mathbf{u}}$ on the bottom surface of the bonding layer $\mathscr{A} \times(-w / 2, w / 2) \times\left\{-h_{b}\right\}$. Assuming that the substrate is infinitely stiff compared to the film, the displacement $\overline{\mathbf{u}}$ may be obtained from the solution of the elastic problem on the substrate under structural loads when neglecting the presence of the film. Inelastic strains $\overline{\mathbf{e}}$ in the film are a second type of loads. They may be defined as the deformations that the film would attain if left free from the substrate and internal compatibility constraints. The inelastic strain may rise in the system as a consequence of thermal loading, drying, or other multi-physical couplings. In the following we assume that $\overline{\mathbf{u}}(\mathbf{x})=\bar{u}(\mathbf{x}) \mathrm{e}_{1}, \overline{\mathrm{e}}(\mathbf{x})=\bar{e}(\mathbf{x}) \mathrm{I}, \mathrm{I}$ being the identity matrix.

\subsection{Energy functional of the one-dimensional model}

We model the system in Figure 1 as an elastic bar on an elastic foundation. We assume that the axis of the film undergoes axial displacement only in the form $\mathbf{u}(\mathbf{x})=u(x) \mathbf{e}_{1}$. Hence, to each displacement field 
$u: x \in \mathscr{A} \mapsto \mathbb{R}$, fracture set $\Gamma$, and debonding set $\Delta$, we associate the following elastic energy:

$$
\mathscr{P}_{t}(u, \Gamma, \Delta):=\int_{\mathscr{A} \backslash \Gamma} \frac{1}{2} K\left(u^{\prime}(x)-\bar{\varepsilon}_{t}\right)^{2} d x+\int_{\mathscr{A} \backslash \Delta} \frac{1}{2} k\left(u-\bar{u}_{t}\right)^{2} d x,
$$

where the loadings $\bar{\varepsilon}_{t}$ and $\bar{u}_{t}$ are parameterized by a single scalar parameter $t$. The first term of the energy represents the elastic energy of the film and the integral excludes regions with transverse cracks across the film. The second term accounts for the elastic energy of the bonding layer and the integral excludes the debonded regions. The constitutive constants $K$ and $k$ in (2) represent the stiffness of the film and the bonding layer. An estimate of their values as a function of the material and geometric parameters of the $3 \mathrm{~d}$ model may be obtained by assuming a state of uniaxial stress in the film and that the bonding layer undergoes pure shear. In this way one obtains:

$$
K=E_{f} h_{f} w, \quad k=\frac{\mu_{b} w}{h_{b}}=\frac{E_{b} w}{2\left(1+v_{b}\right) h_{b}},
$$

where $\mu_{b}=E_{b} / 2\left(1+v_{b}\right)$ is the shear modulus of the bonding layer. Adopting a Griffith model for the fracture energy, we associate to the cracks the surface energy

$$
\mathscr{S}(\Gamma, \Delta):=G_{\Gamma} \#(\Gamma)+G_{\Delta} \mathscr{L}(\Delta)
$$

where $\#(\Gamma)$ is the number of transverse cracks and $\mathscr{L}(\Delta)$ is the total length of the debonded regions. The constants $G_{\Gamma}=G_{f} h_{f} w$ and $G_{\Delta}=G_{b} w$ are the energy required to create a single transverse crack and the debonding energy per unit of length, respectively.

The total energy is

$$
\mathscr{E}_{t}(u, \Gamma, \Delta):=\mathscr{P}_{t}(u, \Gamma, \Delta)+\mathscr{S}(\Gamma, \Delta) .
$$

Without loss of generality we may consider the case $\bar{u}_{t}=0$. This may be easily seen by applying the change of the variable $u^{*}(x)=u(x)-\bar{u}_{t}(x)$ and $\bar{\varepsilon}_{t}^{*}(x)=\bar{\varepsilon}_{t}(x)-\bar{u}_{t}^{\prime}(x)$. In the sequel we focus on the homogeneous case, for which all the material constant and the loading $\bar{\varepsilon}$ are constant in space and $\bar{u}=0$.

Remark 1 The derivation of the one-dimensional energy above as the limit of a three-dimensional energy when $h_{b}$ and $h_{f}$ approach 0 can be rigorously obtained -under suitable scaling hypothesis on the thicknesses and material properties- by $\Gamma$-convergence. The proof is technical by nature and beyond the scope of this paper but will be presented in a future work.

\subsection{Dimensional analysis}

Introducing a length scale $x_{0}$ and a scale for the displacements $u_{0}$, we are able to define the dimensionless variables $x^{*}:=x / x_{0}, u^{*}:=u / u_{0}$ and denoting by $\mathscr{A}^{*}, \Gamma^{*}$ and $\Delta^{*}$ the corresponding rescaled axis and crack sets, one finds

$$
\frac{\mathscr{E}_{t}\left(u^{*}, \Gamma^{*}, \Delta^{*}\right)}{k u_{0}^{2} x_{0}}=\frac{K}{2 k x_{0}^{2}} \int_{\mathscr{A}^{*} \backslash \Gamma^{*}}\left(u^{* \prime}\left(x^{*}\right)-\frac{\bar{\varepsilon}_{t} x_{0}}{u_{0}}\right)^{2} d x^{*}+\int_{\mathscr{A}^{*} \backslash \Delta^{*}} \frac{u^{*}(x)^{2}}{2} d x+\frac{G_{\Gamma}}{k x_{0} u_{0}^{2}} \#\left(\Gamma^{*}\right)+\frac{G_{\Delta}}{k u_{0}^{2}} \mathscr{L}\left(\Delta^{*}\right) .
$$

By choosing the free scaling parameters $x_{0}$ and $u_{0}$ as $x_{0}=\sqrt{\frac{K}{k}}, u_{0}=\frac{\sqrt{G_{\Gamma}}}{\sqrt[4]{K k}}$ one obtains

$$
\mathscr{E}_{t}^{*}\left(u^{*}, \Gamma^{*}, \Delta^{*}\right):=\int_{\mathscr{A}^{*} \backslash \Gamma^{*}} \frac{1}{2}\left(u^{* \prime}\left(x^{*}\right)-\bar{\varepsilon}_{t}^{*}\right)^{2} d x^{*}+\int_{\mathscr{A}^{*} \backslash \Delta^{*}} \frac{1}{2} u^{*}(x)^{2} d x^{*}+\#\left(\Gamma^{*}\right)+\gamma \mathscr{L}\left(\Delta^{*}\right),
$$

with

$$
\bar{\varepsilon}_{t}^{*}=\bar{\varepsilon}_{t} \sqrt[4]{\frac{K^{3}}{G_{\Gamma}^{2} k}}, \quad \gamma=\sqrt{\frac{K}{k}} \frac{G_{\Delta}}{G_{\Gamma}},
$$

and $\mathscr{E}_{t}^{*}:=\mathscr{E}_{t} / G_{\Gamma}$. Three dimensionless parameters determine uniquely the energy function (6): the dimensionless length of the bar $L^{*}=\mathscr{L}(\mathscr{A}) / x_{0}$, the relative bonding toughness $\gamma$, and the loading intensity $\bar{\varepsilon}_{t}^{*}$. Henceforth we will use the non dimensional form of the energy (6) but we will drop the ${ }^{*}$ for the sake of conciseness. 
3.4 Quasi-static evolution problem

We consider a film free at its ends. For a given transverse fracture set $\Gamma$, the set of admissible displacement fields is independent of the debonded region $\Delta$ and belongs to the space $\mathscr{C}(\Gamma)=H^{1}(\mathscr{A} \backslash \Gamma)$ of square integrable displacement fields with square integrable first derivatives on $\mathscr{A} \backslash \Gamma$. The displacements may be discontinuous across the transverse cracks $\Gamma$.

In this context, we start giving the following definition of quasi-static evolution, which is common in the variational theory of rate independent processes [21,18].

Definition 1 (Quasi-static evolution) Given a loading path $\bar{\varepsilon}_{t}$ for $t \in\left[0, t_{\max }\right]$, a function $t \rightarrow\left(u_{t} \in \mathscr{C}\left(\Gamma_{t}\right), \Gamma_{t} \subset\right.$ $\mathscr{A}, \Delta_{t} \subseteq \mathscr{A}$ ) is a quasi-static evolution (or "the solution of the quasi-static evolution problem") if it satisfies the Continuous Evolution Law (CEL), given by the following items:

(IR) Irreversibility of the crack evolution: The functions $\left(\Gamma_{t}, \Delta_{t}\right)$ must be non-decreasing with $t$, more precisely:

$$
\Gamma_{t} \supseteq \Gamma_{s}, \quad \Delta_{t} \supseteq \Delta_{s}, \quad \forall 0 \leq s \leq t .
$$

(GST) Unilateral global stability: At every time $t$ the state $\left(u_{t} \in \mathscr{C}_{t}\left(\Gamma_{t}\right), \Gamma_{t}, \Delta_{t}\right)$ is the global minimizer of the total energy among all admissible states:

$$
\mathscr{E}_{t}\left(u_{t}, \Gamma_{t}, \Delta_{t}\right) \leq \mathscr{E}_{t}(u, \Gamma, \Delta), \quad \forall u \in \mathscr{C}_{t}(\Gamma), \quad \forall \Gamma \supseteq \Gamma_{t}, \quad \forall \Delta \supseteq \Delta_{t}
$$

(EB) Energy balance: The function $E(t):=\mathscr{E}_{t}\left(u_{t}, \Gamma_{t}, \Delta_{t}\right)$ is absolutely continuous in $t$ and satisfies:

$$
E(t)-E(0)=-\int_{0}^{t} \int_{\mathscr{A}} \sigma_{t} \frac{d \bar{\varepsilon}_{t}}{d t} d x d t
$$

where $\sigma_{t}:=K\left(u_{t}^{\prime}(x)-\bar{\varepsilon}_{t}\right)$.

We choose to study Monotonically Increasing Loads (MIL), more precisely, throughout the whole work, we focus on homogeneous proportional loads of the type

$$
\bar{\varepsilon}_{t}=t
$$

\section{Solution of the static problem: energy minimizers at fixed load}

Before looking for the solutions of the evolution problem, we study the crack states that are energy minimizers for a given loading, without involving any notion of history or irreversibility. We refer to this problem as the static problem. Formally it consists, for a given loading parameter $t$, in finding $u_{t} \in \mathscr{C}\left(\Gamma_{t}\right), \Gamma_{t} \in \mathscr{A}, \Delta_{t} \in \mathscr{A}$ that verifies the condition below:

(GM) Global minimality:

$$
\mathscr{E}_{t}\left(u_{t}, \Gamma_{t}, \Delta_{t}\right) \leq \mathscr{E}_{t}(u, \Gamma, \Delta), \quad \forall u \in \mathscr{C}_{t}(\Gamma), \quad \forall \Gamma \in \mathscr{A}, \forall \Delta \subseteq \mathscr{A}
$$

Remark 2 The condition (GM) given in Equation (12) is stronger that the condition (GST) given in Equation (9). In (GST), the admissible transverse cracks and debonded sets must satisfy the irreversibility conditions $\Gamma \supseteq \Gamma_{t}, \Delta \supseteq \Delta_{t}$.

In the sequel, we focus first on the purely elastic case (without transverse cracks or debonding), on the uncoupled cases (transverse cracks only, then debonding only), an finally on the fully coupled model. 
4.1 Elastic solution

We look for the elastic solution for a sound film, i.e. the displacement field that verifies $(12)$ for $\Delta=\Gamma=\emptyset$. The solution is unique because of the convexity of the elastic energy (linear elasticity). It must belong to $\mathscr{C}(\emptyset)$ and satisfy the the first order local minimality condition:

$$
D_{u} \mathscr{E}(u, \emptyset, \emptyset)(v)=\int_{\mathscr{A}}\left(\left(u^{\prime}(x)-t\right) v^{\prime}(x)+u(x) v(x)\right) d x=0, \quad \forall v \in \mathscr{C}(\emptyset),
$$

where $D_{u} \mathscr{E}(u, \cdot, \cdot)(v)$ denotes the Gateaux derivative of the functional $\mathscr{E}(u, \cdot, \cdot)$ with respect to $u$ along the direction $v$. After integration by parts, and applying standard arguments of the Calculus of Variation, one obtains that 13 implies:

$$
-u^{\prime \prime}(x)+u(x)=0 \quad \forall x \in(-L / 2, L / 2), \quad u^{\prime}(-L / 2)=u^{\prime}(L / 2)=t,
$$

which are the strong formulation of the equilibrium equations plus the natural boundary conditions. They may be integrated to get

$$
u_{t}(x)=t \frac{\sinh (x)}{\cosh (L)}
$$

The elastic displacement field is plotted in Figure 2. The total energy corresponding to this solution is

$$
E_{t}(L):=\mathscr{E}\left(u_{t}, \emptyset, \emptyset\right)=t^{2} F(L), \quad \text { with } \quad F(L)=\left(\frac{L}{2}-\tanh \left(\frac{L}{2}\right)\right) .
$$
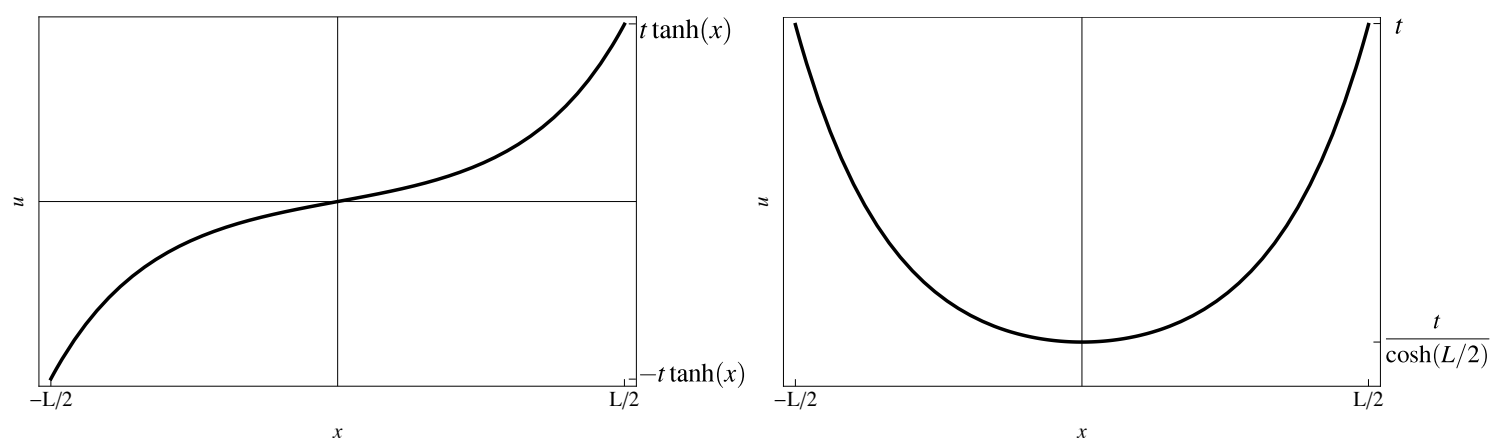

Fig. 2 Elastic displacement (left) and deformation (right) for a sound film of dimensionless length $L=6$

4.2 Solution for a perfectly bonded film subject to transverse cracks only

We now assume that transverse cracks are free to appear everywhere, but debonding is proscribed $(\Delta=\emptyset)$. The topology of the cracks in the one dimensional setting is simple. A general state with $n-1$ cracks is sketched in Figure 3 . The domain $\mathscr{A}$ is split into $n$ parts, the crack set being the set of $n-1$ points $\Gamma^{(n)}=$ $\left\{-L / 2<x_{i}<L / 2\right\}_{i=1}^{n-1}$.

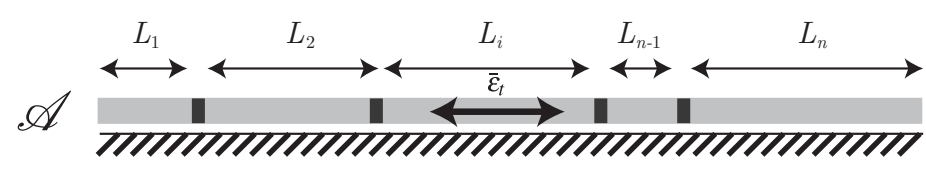
$-\mathrm{L} / 2$
$x_{i}$
$x_{i+1}$
$\mathrm{L} / 2$

Fig. 3 A $1 D$ film split into $n$ pieces thus with $n-1$ transverse cracks. 


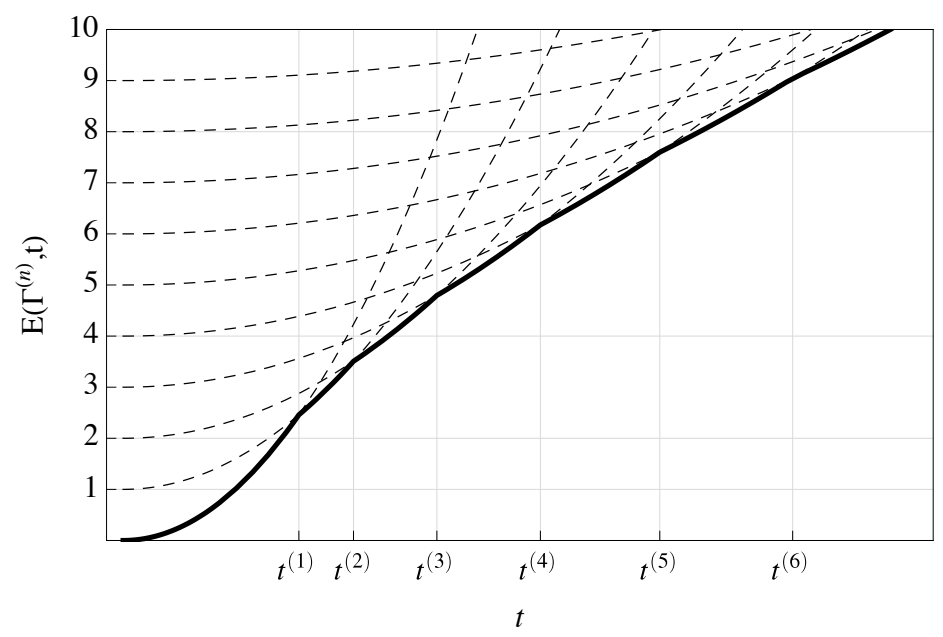

Fig. 4 Total energy for transverse fracture without debonding for different number of cracks. The cracks are equally spaced. The optimal crack number for a given loading is that of the curve attaining the lowest energy level, this last indicated by the continuous stroke.

Each part can be seen as a sound domain of (dimensionless) length $L_{i}=x_{i}-x_{i-1}$, where $x_{0}=-L / 2$ and $x_{n}=L / 2$. Hence, using the results of section 4.1 and equation (16), the total energy of the system may be written as the following function of the number of parts $n$ and the length of each part $L_{i}$ :

$$
E_{t}\left(n ; L_{1}, \ldots, L_{n}\right)=t^{2} \sum_{i}^{n} F\left(L_{i}\right)+(n-1) .
$$

For a fixed number of crack $n$, finding the optimal crack lengths $L_{1}, \ldots, L_{n}$ according to 12 consists in solving the following minimization problem

$$
\min _{L_{1}, \ldots, L_{n}} E_{t}\left(n ; L_{1}, \ldots, L_{n}\right), \quad \text { with } \sum_{i=1}^{n} L_{i}=L, \quad L_{i}>0,
$$

which is an optimization problem for a function of $n$ scalar variables with linear constraints. Observing that $\partial^{2} E_{t} / \partial L_{i} \partial L_{j}=\delta_{i j} t^{2} F^{\prime \prime}\left(L_{i}\right)$, where $\delta_{i j}=1$ for $i=j$ and $\delta_{i j}=0$ for $i \neq j$, and being

$$
F^{\prime \prime}\left(L_{i}\right)=4 \sinh \left(L_{i} / 2\right)^{4} / \cosh \left(L_{i}\right)^{3}>0, \forall L_{i}>0,
$$

we conclude that the energy is a strictly convex function of $L_{i}$. Hence the minimization problem (18) admits a unique solution, which is given by

$$
L_{i}=\frac{L}{n}, \quad \forall i=1, \ldots, n .
$$

This shows that the optimal crack arrangement is that of an equally distributed array of cracks throughout the film, recovering experimental evidence of periodicity of the cracks [22], without making any a priori assumption on the topology of the crack set.

The total energy of a system with $n-1$ equally spaced transverse fractures is:

$$
E_{t}^{(n)}=t^{2} \sum_{i}^{n} F(L / n)+(n-1) .
$$

In Figure 4, we plot $E_{t}^{(n)}$ as a function of $t$ for different values of $n$. By (GM), the optimal number of cracks for a given load is that of the curve attaining the lowest energy. The corresponding critical loadings $t^{(n)}$ in Figure 4 may be found analytically by looking for the intersections between $E_{t}^{(n)}$ and $E_{t}^{(n+1)}$. Hence we conclude with the following result. 
Proposition 1 The solution of the problem (12) without debonding is that of an equally spaced array of cracks partitioning the film into $n$ regular segments of length $L_{i}=L / n$. The displacement in each regular segment is in the form (15) with $L=L_{i}$ and the total energy of the system is given by (20). The solution with $n-1$ cracks is optimal for

$$
t^{(n-1)}<t<t^{(n)}, \quad \text { with } \quad t^{(n)}=\frac{1}{\sqrt{(1+n) \tanh \frac{L}{2(1+n)}-n \tanh \frac{L}{2 n}}}, \quad t^{(0)}:=0 .
$$

Remark 3 (A limit model for long films) When the film is very long it may be consistent to describe the system in terms of a "fracture density" rather than by the absolute number of fractures. In such setting, fracture density may be thought of as a macroscopic characterization of a damaging process. A limit model for long films is deduced from the total energy of Equation (20) replacing the discrete variable $n$ with the continuous density of fractures defined by $\alpha:=n / L$. Minimizing the energy $(20)$ seen as a function of $\alpha$ for $\alpha>0$, we recover the envelope of the family of energies $E_{t}^{(n)}, n \in \mathbb{N}$ as Figure 5 shows. The total energy for the limit model is displayed in Figure 5(a) with a black solid line, enveloping the family of energies $\bar{E}_{t}^{(n)}$. In Figure 5(b) we compare the discrete number $n_{t}$ of cracks with the crack density $\alpha_{t}$, in the long film regime. Both figures relate to a film of dimensionless length $L=20$. Note that long films with transverse cracks can be regarded as an example of continua with structured deformations and modelled within the framework developed in [14].

\subsection{Solution for a perfectly elastic film subject to debonding only}

Introducing the characteristic function of the debonded part of the domain $\Delta$ defined as

$$
\chi_{\Delta}(x): x \in \mathscr{A} \mapsto\{0,1\}, \quad \chi_{\Delta}(x)= \begin{cases}1, & \text { if } x \in \Delta \\ 0, & \text { otherwise }\end{cases}
$$

the total energy (6) is rewritten as follows

$$
\tilde{\mathscr{E}}_{t}\left(u, \Gamma, \chi_{\Delta}\right):=\int_{\mathscr{A} \backslash \Gamma}\left(\frac{1}{2}\left(u^{\prime}(x)-t\right)^{2}+\frac{1}{2} u(x)^{2}\left(1-\chi_{\Delta}\right)+\gamma \chi_{\Delta}\right) d x+\#(\Gamma) .
$$

The energy density, for a fixed point $x \in \mathscr{A}$ is linear in $\chi_{\Delta}$. The optimality with respect to $\chi_{\Delta}$ gives a local debonding condition depending on the value of $2 \gamma / u(x)^{2}$, namely:

$$
\chi_{\Delta}(x)=\left\{\begin{array}{ll}
1, & \text { if }|u(x)|<u_{c} \\
0, & \text { if }|u(x)| \geq u_{c}
\end{array},\right.
$$

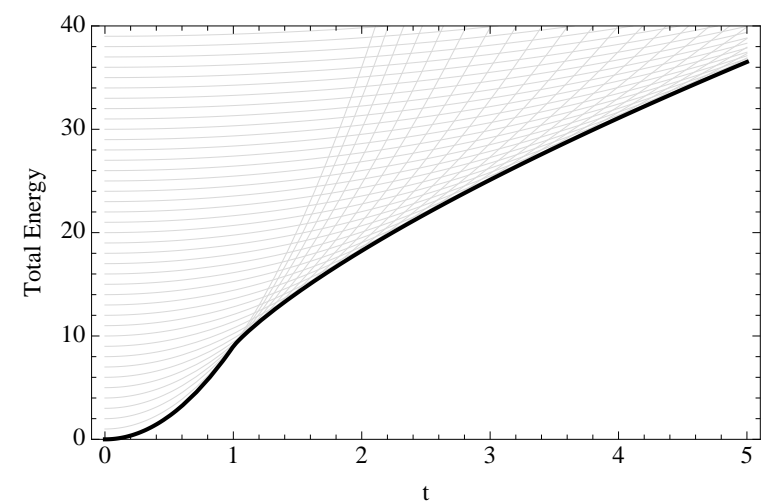

(a)

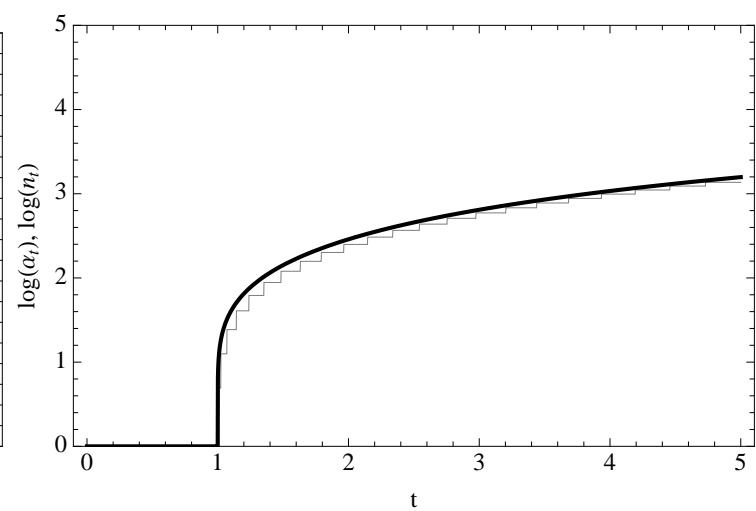

(b)

Fig. 5 Energy and fracture density curves in the "long film" regime. Thick lines show the results of the limit model, they are compared with the discrete family of energies 20. The limit model can macroscopically characterize high fracture density regimes with a "damage"-like parameter. 
where $u_{c}=\sqrt{2 \gamma}$ is the critical displacement threshold for debonding. The elimination of $\chi_{\Delta}$ according to 23. allows us to rewrite the energy, and thus reformulate the problem, in terms of $u$ and $\Gamma$ alone, as follows:

$$
\tilde{\mathscr{E}}_{t}(u, \Gamma):=\frac{1}{2} \int_{\mathscr{A} \backslash \Gamma}\left(u^{\prime}(x)-t\right)^{2}+f(|u(x)|) d x+\#(\Gamma)
$$

where we introduced the non-smooth, non-convex energy density

$$
f(\delta)= \begin{cases}\delta^{2}, & \text { if } \delta<u_{c}=\sqrt{2 \gamma} \\ \gamma, & \text { if } \delta \geq u_{c}=\sqrt{2 \gamma}\end{cases}
$$

which accounts for both the elastic contribution of the bonding layer and the surface energy related to debonding. The energy density in (24) is non-convex with respect to $u$ and not differentiable in $u$ at the critical threshold $u_{c}$. According to the global minimality principle (GM), the problem of finding the solution with debonding and without transverse fractures (i.e. $\Gamma=\emptyset$ ) is formulated as follows:

$$
\min _{u \in \mathscr{C}_{t}(\emptyset)} \tilde{\mathscr{E}}_{t}(u, \emptyset)
$$

Unlike in the purely elastic case (Section 4.1), here the solution for the displacement cannot be derived using the Euler-Lagrange equations associated to the first order local minimality condition for $\tilde{\mathscr{E}}_{t}(u, \emptyset)$, because of the non-differentiability of the energy density for $u=u_{c}$. However, the local minimizers of $\tilde{\mathscr{E}}_{t}(u, \emptyset)$ are characterized by the following fundamental properties, whose proofs are given in Appendix. Note that the present problem and the results below are very similar to those presented in [23].

Lemma 1 If a field $u \in \mathscr{C}_{t}(\emptyset)$ is a local minimum of $\tilde{\mathscr{E}}_{t}(u, \emptyset)$, then $u$ is a monotonic function of $x$.

Lemma 2 Let $f: \mathbb{R} \mapsto \mathbb{R}$ be a continuous function. If a field $u \in \mathscr{C}_{t}(\emptyset)$ is a local minimum of $\tilde{\mathscr{E}}_{t}(u, \emptyset)$, it satisfies the following first integral and boundary conditions:

$$
\begin{aligned}
\exists C \in \mathbb{R}: & u^{\prime}(x)^{2}-t^{2}-f(|u(x)|)=C, \quad \forall x \in[0, L], \\
& u^{\prime}(-L / 2)=u^{\prime}(L / 2)=t .
\end{aligned}
$$

The monotonicity of the solution implies that the maximal values of $|u|$ are attained at the ends of the domain. Hence, in view of the debonding condition (23), if debonding takes place, the non-debonded part of the domain is an interval. and the debonded domain is of the form:

$$
\Delta=\left[-L / 2,-L / 2+D_{1}\right] \cup\left[L / 2-D_{2}, L / 2\right], \quad \text { with } \quad D_{1}, D_{2} \geq 0, D:=D_{1}+D_{2} \leq L .
$$

Using Equations (26), one may compute explicitly the solution with debonding, which is in the form (see Figure 6(b)

$$
u(x)= \begin{cases}t\left(x+L / 2-D_{1}+\tanh ((L-D) / 2)\right), & -L / 2 \leq x \leq-L / 2+D_{1} \\ t \frac{\sinh \left(x-x_{0}\right)}{\cosh ((L-D) / 2)}, & -L / 2+D_{1} \leq x \leq L / 2-D_{2} \\ t\left(x-L / 2+D_{2}+\tanh ((L-D) / 2)\right), & -L / 2-D_{2} \leq x \leq L / 2\end{cases}
$$

where $x_{0}=\left(D_{1}-D_{2}\right) / 2$ is the coordinate of the center of the bonded domain. In particular, the debonded regions are stress free and the elastic energy vanishes there. Hence, denoting by $D$ the total debonded length and $B:=L-D$ the length of the bonded interval, the total energy of the system is a function of $B$ alone and reads as

$$
\tilde{E}_{t}(B):=E_{t}(B)+\gamma(L-B)=t^{2} F(B)+\gamma(L-B)
$$

where $E_{t}$ is given by $(16)$. For a fixed loading $t$, the optimal size of the debonded region solves the following problem:

$$
\min _{0 \leq B \leq L} \tilde{E}_{t}(B)
$$


which is the minimization of a strictly convex function of $B$ with inequality constraints and admits a unique solution for the optimal bonded length $0 \leq B^{*} \leq L$. This solution has to satisfy the following first order local minimality condition

$$
\tilde{E}_{t}^{\prime}\left(B^{*}\right)\left(B-B^{*}\right) \geq 0, \quad \forall 0 \leq B \leq L, \quad \text { with } \quad \tilde{E}_{t}^{\prime}(B)=t^{2} \tanh ^{2}(B / 2) / 2-\gamma .
$$

By the properties of convex functions, the unique minimum is attained in $B^{*}=0$ if and only if $E^{\prime}(0) \geq 0$, in $B^{*}=L$ if and only if $E^{\prime}(L) \leq 0$, and in $0<B^{*}<L$ such that $\tilde{E}_{t}^{\prime}\left(B^{*}\right)=0$ otherwise. The first case $B^{*}=0$ in impossible because $\tilde{E}_{t}^{\prime}(0)=-\gamma<0$. The second case $B^{*}=L$ corresponds to a solution without debonding. It is obtained if and only if $t \leq \sqrt{2 \gamma}$ or $L \leq 2 \operatorname{arctanh} \sqrt{2 \gamma / t^{2}}$. For $t>\sqrt{2 \gamma}$ and $L>2 \operatorname{arctanh} \sqrt{2 \gamma / t^{2}}$, the solution is such that $\tilde{E}_{t}^{\prime}\left(B^{*}\right)=0$, that is $B^{*}=2 \operatorname{arctanh} \sqrt{2 \gamma / t^{2}}$. This latter case corresponds to solutions with debonding.

We resume the results of the debonding of a film of length $L$ without transverse fracture in the following Proposition:

Proposition 2 For a fixed loading $t$, the solution of the problem (GM) with debonding and without transverse fractures $(\Gamma=\emptyset)$ is in the form (28). The displacement field $u$ is a monotonic function of $x$, the bonded domain is an interval and debonding, if present, takes place at the ends of the domain. The debonded domain $\Delta$ has the form (27). The total debonded length $D$ is uniquely determined, but the lengths $D_{1}$ and $D_{2}$ are arbitrary. The optimal length of the bonded interval is

$$
B_{t}(L)= \begin{cases}L & \text { for } \quad L \leq L_{b}(t, \gamma) \\ L_{b}(t, \gamma) & \text { for } L>L_{b}(t, \gamma)\end{cases}
$$

where

$$
L_{b}(t, \gamma):=\left\{\begin{array}{ll}
\infty & \text { for } t \leq t_{c}=\sqrt{2 \gamma} \\
2 \operatorname{arctanh} \sqrt{2 \gamma / t^{2}}, & \text { for } t>t_{c}
\end{array},\right.
$$

In particular, for $t \leq t_{c}=\sqrt{2 \gamma}$ there is no debonding, independently of the film length. For $t>t_{c}$ the film debonds if and only if it is suffciently long $\left(L>L_{b}(t, \gamma)\right)$ or, equivalently, the load is sufficient high, i. e. $t>t_{b}(L, \gamma)$, with

$$
t_{b}(L, \gamma):=\sqrt{2 \gamma} \operatorname{coth} \frac{L}{2}
$$

The energy of the optimal solution is

$$
\hat{E}_{t}(L):=\tilde{E}_{t}\left(B_{t}(L)\right)=\left\{\begin{array}{ll}
t^{2}(L / 2-\tanh (L / 2)) & \text { for } \quad L \leq L_{b}(t, \gamma) \\
\gamma L+2 t^{2}\left(\left(1-2 \gamma / t^{2}\right) \operatorname{arctanh} \sqrt{2 \gamma / t^{2}}-\sqrt{2 \gamma / t^{2}}\right) & \text { for } \quad L>L_{b}(t, \gamma)
\end{array} .\right.
$$

The main results are graphically illustrated in Figure 6, which shows a phase diagram for the bonded length $B$ as a function of $(L, t)$, and a snapshot of the displacement field for a partially bonded film.

\subsection{Solution for a film subject to coupled transverse cracks and debonding}

We now consider the case in which transverse fracture and debonding may take place at the same time in a film of length $L$. Suppose that the film is subdivided by $n-1$ transverse fractures in $n$ segments of lengths $\left\{L_{1}, \ldots, L_{n}\right\}$. Given the segment length $L_{i}$, the solution for the displacement in the $i$-th segment is that of a film with possible debonding and without transverse fracture, which is resumed in Proposition 2 Hence, the total energy of the system may be written as follows:

$$
\hat{E}_{t}\left(n ; L_{1}, \ldots, L_{n}\right):=\sum_{i}^{n} \hat{E}_{t}\left(L_{i}\right)+(n-1)
$$




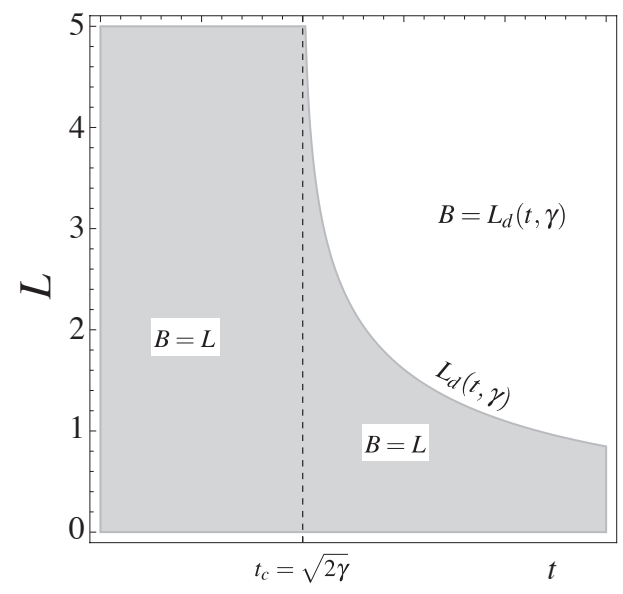

(a)

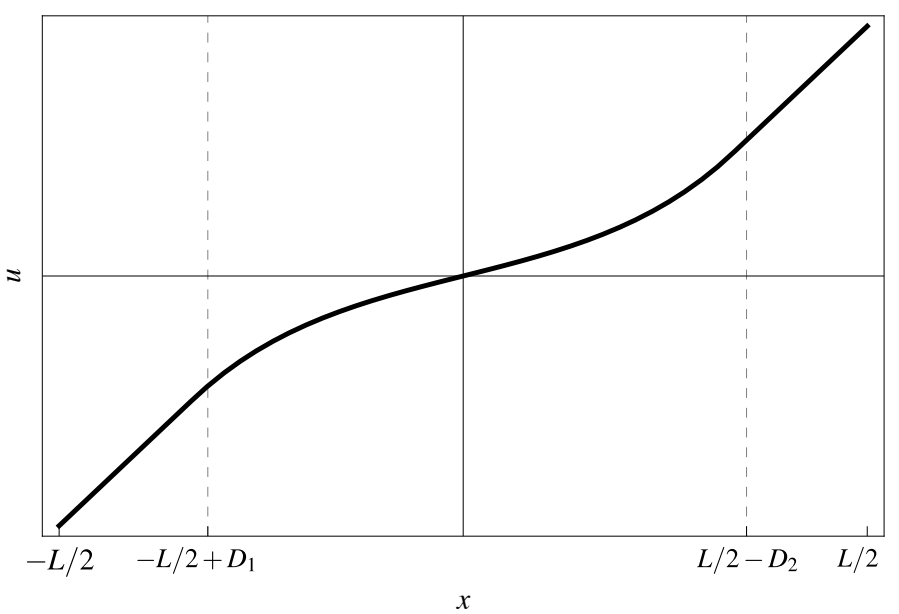

(b)

Fig. 6 Solution of (GM) for a film with debonding and without transverse fracture. Figure 6(a) phase diagram of the optimal size of the bonded domain, as function of the film length and load intensity, where $L_{b}(t, \gamma)$ is given in Equation (33). Figure 6(b) displacement field for the partially bonded film, see Equation [28.

where $\hat{E}_{t}\left(L_{i}\right)$, defined in equation $(35)$, is the sum of the elastic and the debonding energy of the $i$-th segment. As in Section 4.2, for a fixed number of transverse cracks, the problem of finding the optimal segment lengths $L_{i}$ 's may be formulated as the following constrained optimization problem:

$$
\min _{L_{1}, \ldots, L_{n}} \hat{E}_{t}\left(n ; L_{1}, \ldots, L_{n}\right), \quad \text { with } \sum_{i=1}^{n} L_{i}=L, \quad L_{i}>0 .
$$

For $t<t_{c}$ the energy is strictly convex with respect to the $L_{i}$ 's. As in Section 4.2 , the solution is in the form of periodic transverse cracks without delamination, with $L_{i}=L / n$ and the displacement field in each segment is as in equation (15) and Figure 2 (with $L=L_{i}$ ).

For $t>t_{c}$ the energy is not convex anymore with respect to the $L_{i}$ 's. Introducing the Lagrange multiplier $\lambda$ associated to the equality constraint $\sum_{i=1}^{n} L_{i}=L$, the first order optimality conditions for (36) reads as

$$
\hat{E}_{t}^{\prime}\left(L_{i}\right)=\lambda \quad \text { for } \quad i=1 \ldots n \text { and } \quad \sum_{i=1}^{n} L_{i}=L .
$$

The derivative of the energy of a single segment, $\hat{E}_{t}^{\prime}\left(L_{i}\right)$, is strictly monotonic, increasing from 0 to $\gamma$, for $0 \leq L_{i}<L_{b}(t, \gamma)$ and constantly equal to $\gamma$ for $L_{i} / n>L_{b}(t, \gamma)$. Hence, we may classify the solution of (37) in two types as a function of the optimal value of $\lambda$ :

(i) $\lambda<\gamma\left(\right.$ solutions without debonding). In this case $\hat{E}_{t}^{\prime}\left(L_{i}\right)=\lambda$ admits a unique solution, which is the same for each $L_{i}$. All the crack lengths must be the equal and, from $\sum_{i=1}^{n} L_{i}=L$, we find $L_{i}=L / n$. This is the case of periodic cracking without debonding, as in Section 4.2. This solution is admissible only if $L_{i}=L / n \leq L_{b}(t, \gamma)$, i.e. for $t<t_{b}(L / n, \gamma)$, where is the $t_{b}(L, \gamma)$ is the critical load for debonding a film of length $L$ given by Equation 34 .

(ii) $\lambda=\gamma$ (Solutions with debonding). In this case the problem (37) admits infinitely many solutions with $L_{i} \geq L_{b}(t)$. Even if the $L_{i}$ 's are not uniquely determined, being $L_{i} \geq L_{b}(t)$ debonding is necessarily present in each segment. The length of the bonded part in each domain is $B=L_{b}(t, \gamma)$ and the displacement field in each segment is in the form (28) (see Proposition 2). Being $L=\sum_{i=1}^{n} L_{i} \geq n L_{b}(t, \gamma)$, this solution is possible only for $t>t_{b}(L / n, \gamma)$. Note that only the length of the bonded interval of each part is uniquely determined. The lengths $L_{i}$ 's are not uniquely determined because, for each part, all the solutions obtained transferring debonded regions from one end of to the other, without changing the bonded length, are energetically equivalent. 
Hence we may conclude with the following proposition which gives the static solution with $n-1$ transverse fractures and with free debonding as a function of $t$.

Proposition 3 The solution of the problem (GM) for a film of total length L and relative fracture toughness $\gamma$ with possible debonding and with $n-1$ transverse cracks is as follows:

(i) For $t<t_{d}^{(n)}:=t_{b}(L / n, \gamma)$ there is not debonding and the cracks are equally spaced with $L_{i}=L / n$.

(ii) For $t \geq t_{d}^{(n)}$, there is debonding. The solution for the displacement field in each segment of the film is in the form (28) with $L=L_{i}, D_{1}=D_{1, i}$ and $D_{2}=D_{2, i}$. The length of the bonded interval is $L_{b}(t, \gamma)$ in each segment. The total debonded length is $D:=\sum_{i=1}^{n} D_{1, i}+D_{2, i}=L-n L_{b}(t, \gamma)$. However, the length of the debonded parts of each segment are not uniquely determined, because all the solutions obtained by varying $D_{i, 1}, D_{i, 2}$ and keeping constant the total debonded length $D$ have the same energy.

The total energy of the solution is

$$
\hat{E}_{t}^{(n)}:=\left\{\begin{array}{lll}
t^{2}(L / 2 n-\tanh (L / 2 n))+(n-1) & \text { for } t \leq t_{d}^{(n)} \\
(\gamma L-1)+n\left(1-t^{2} \varphi(\sqrt{2 \gamma} / t)\right) & \text { for } t>t_{d}^{(n)}
\end{array}\right.
$$

where $\varphi(x)=x+\left(x^{2}-1\right) \operatorname{arctanh}(x)$.

In order to close the problem, we must minimize $\hat{E}_{t}^{(n)}$ for a given load $t$. We are not able to provide a complete analytical solution of the latter, however we illustrate a typical scenario for specific values of the numerical parameters with the help of the energy plot. In Figure 7 we show the total energy $v s t$ for different values of $n$, for the coupled transverse fracture-debonding problem for $\gamma=2.2, L=6$. The right figure is a zoom on the gray shaded region. We can identify each of the curves with the corresponding value of $n$ noticing that for a vanishing load, the energy reduces to the number of cracks $n-1$. The dashed lines in the figure distinguished the states in which debonding has been activated. We reconstruct the optimal state of the system by comparing the energy levels in Figure 7. For $t<t_{d}^{(4)}$ the optimal solution is with transverse fracture without debonding. The critical loads $t^{(n)}$, defining the range of optimality for a solution with $n-1$ cracks, are those defined by Equation (21). Moreover, there exists a critical load $t^{*}$ beyond which the optimal solution is always that of a single delaminated segment of length $L_{b}(t, \gamma)$ given by Equation (33). Indeed, for $t>t^{*}$ the lowest energy is always attained with $n=1$. Such critical load is the (unique) solution of the equation: $1-t^{2} \varphi(\sqrt{2 \gamma} / t)=0$, where $\varphi(x)$ has been defined in Proposition 3 . The critical time $t^{*}$ is indicated in Figure 7 with a solid vertical line. The range of loading parameter for which there is a true coupling between debonding and transverse fracture is $t^{(4)} \leq t \leq t^{*}$. To better illustrate the solution, we report in Figure 8 the optimal displacement field for the following three representative loadings:

$-t=3.00$, Figure 8(a) the minimum of the energy is attained on the energy curve relative to $n=4$, at the point $\mathrm{A}$ in Figure 7. The load is below the debonding threshold $t_{d}^{(4)}=3.22$ associated to $n=4$. The film is in the periodic multifissuration regime, with three transverse fractures.

$-t=3.30$, Figure $8(\mathrm{~b})$ the minimum energy is attained at the point $B$ of Figure 7 on the energy branch for which debonding is active (dashed line). The energy curve is associated to $n=4$, and the value of the bonded domain size, for each of the four segments into which the film is split is $B_{t}=1.16$.

$-t=4.51$, Figure $8(\mathrm{c})$ the global energy minimizer is the state without transverse fractures denoted by the point $\mathrm{C}$ in Figure 7 The film is bonded on a domain of size $B_{t}=1.08$.

This result may be extended to generic values of $L$ and $\gamma$ as follows.

Proposition 4 For a film of length $L$ and relative debonding toughness $\gamma$, let $\bar{n}$ be the smallest (positive integer) value of $n$ for which $t_{d}^{(n)}<t^{(n+1)}$ and let $t^{*}$ be the (unique) root of $1-t^{2} \varphi(\sqrt{2 \gamma} / t)=0$. Then the solution of the static problem (GM) with free transverse fracture and debonding is in the following form:

- For $t<t_{d}^{(\bar{n})}$, it is with transverse fractures only, with $n-1$ equally spaced cracks in each loading interval $t^{(n-1)}<t<t^{(n)}$ with $n \leq \bar{n}$, as in Proposition 1 .

- For $t>t^{*}$ it is with debonding only, as in Proposition 2.

The number $\bar{n}$ is the minimum number of parts in which a film is split by transverse fractures when there is debonding. The dependence of $\bar{n}$ and $t^{*}$ on the the two dimensionless parameters $L$ and $\gamma$ is illustrated numerically in Figure 9. The critical load $t^{*}$ is independent of $L$. 
For loadings in the interval $t_{d}^{(\bar{n})}<t<t^{*}$, there is a non-trivial coupling between transverse fracture and debonding and we cannot derive a general and simple result of what can happen for generic values of $\gamma$ and $L$.

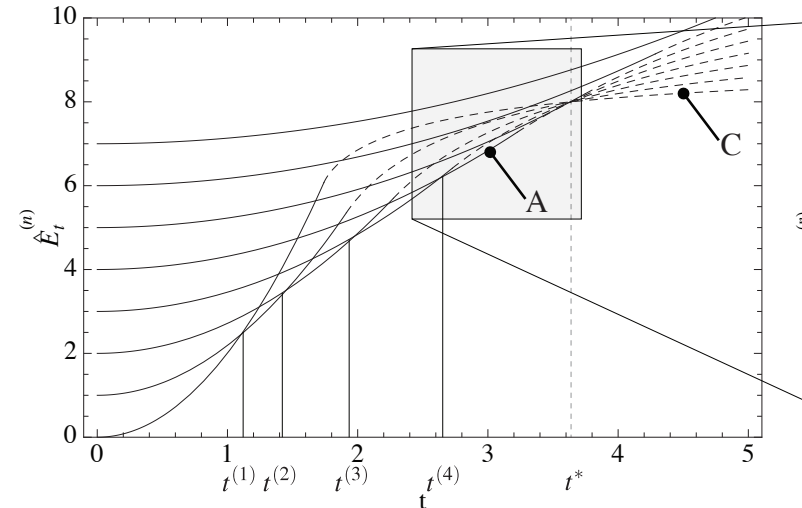

(a)

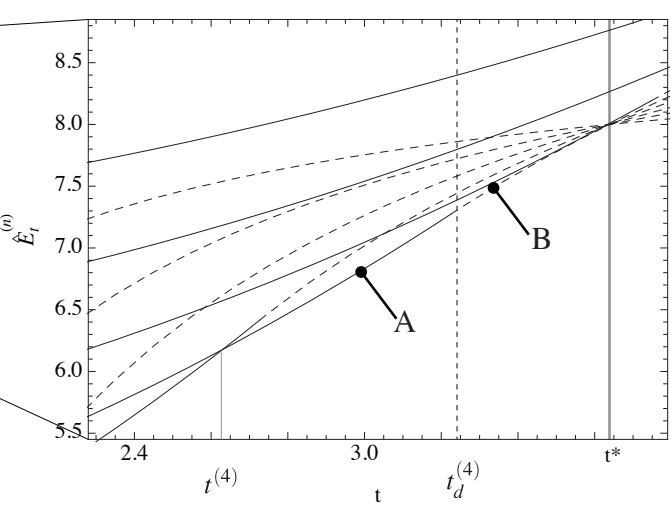

(b)

Fig. 7 Energy curves $\hat{E}_{t}^{(n)}$ with possible debonding and transverse fracture for $\gamma=2.2, L=6$. Each curve is for a specific number $n-1$ of transverse fractures, corresponding to the value at the intersection with the axis $t=0$. The solid continuous lines indicate the energy is obtained for a state without debonding, the dashed line for a state with debonding.

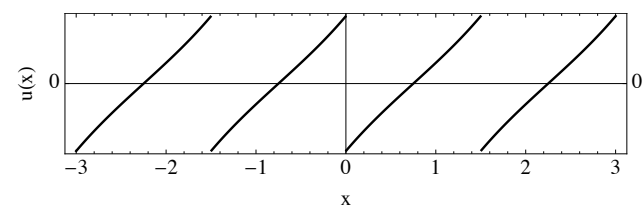

(a)

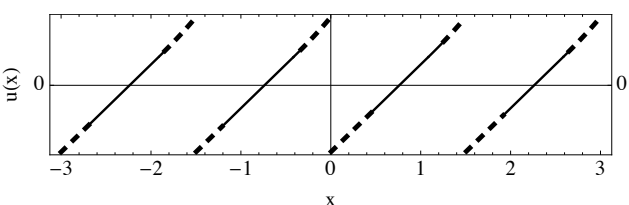

(b)

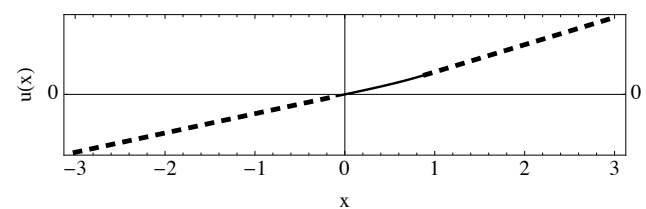

(c)

Fig. 8 The optimal displacement fields for the states A, B, C of Figure 7 Debonded regions are indicated with a dashed line.

\section{The Time-Continuous Quasi-Static Evolution}

We illustrate now some of examples of time-continuous evolutions $t \rightarrow\left(u_{t} \in \mathscr{C}\left(\Gamma_{t}\right), \Gamma_{t} \in \mathscr{A}, \Delta_{t} \in \mathscr{A}\right)$ satisfying the items (IR), (GST) and (EB) under a Monotonically Increasing Load (MIL) of the type of Equation (11). We will take as initial state at load $t=0$ the uncracked solution $(u=0, \Gamma=\emptyset, \Delta=\emptyset)$. The timecontinuous evolutions are constructed on the basis of the results of the static analysis of the previous Section, by using the fact that all the static solutions a fortiori verify the (GST) condition (see the remark at the beginning of Section 4). The (EB) condition is met by imposing the continuity of the total energy with respect to the load $t$, a requirement which is verified also by the static solutions reported in Figures 4, 5, and 7, A major novelty will be the introduction of the irreversibility condition (IR) of Equation 8. 


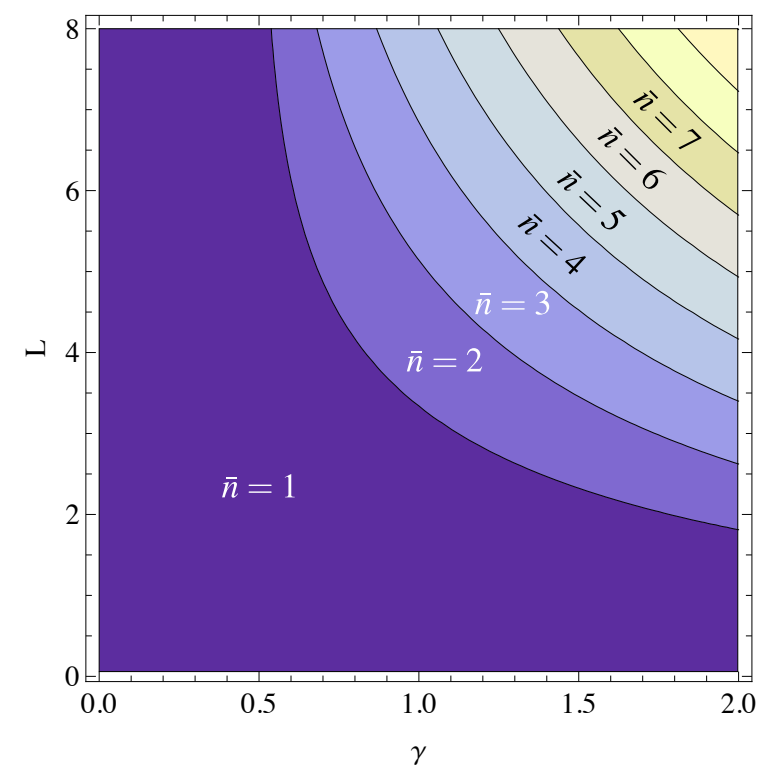

(a)

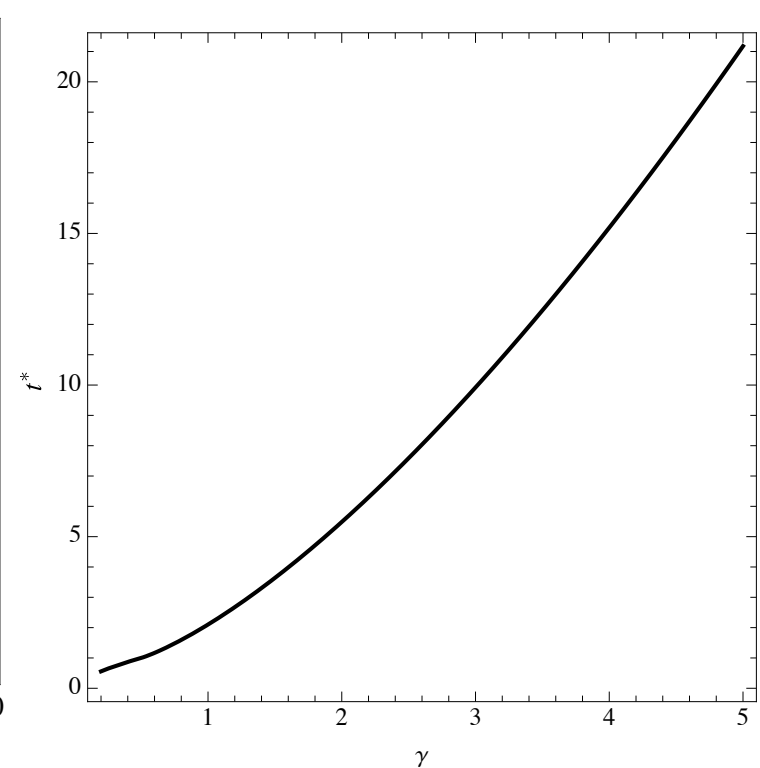

(b)

Fig. 9 Key properties of the solution of the static problem of a film of dimensionless length $L$ and relative debonding toughness $\gamma$ (see Proposition 4). (a) Plot of $\bar{n}$ as a function of $L$ and $\gamma, \bar{n}$ being the minimum number of pieces into which the film is split by transverse fractures when debonding appear (b) Plot of $t^{*}$ as a function of the relative debonding toughness $\gamma, t^{*}$ being the critical load beyond which the optimal solution is that of a debonded film without transverse fractures ( $t^{*}$ is independent of the dimensionless length $L$ of the film).

\subsection{Perfectly bonded film subject to transverse cracks only}

In the case without debonding $(\Delta=\emptyset)$, the static analysis of Section 4.2 (Proposition 1) concludes that the state with $n-1$ cracks partioning the domain in $n$ regions of length $L / n$ is optimal in the sense of the global stability condition (GST) for the load $t$ in the interval $\left(t^{(n-1)}, t^{(n)}\right)$, given by Equation (21). The critical loads of Equation (21) are a strictly increasing sequence with respect to $n$, thus for an initially sound film, the first critical load is $t^{(2)}$ and failure consists in one add-crack in the middle of the film. After the first crack, the irreversibility condition (IR) imposes a restriction on the admissible crack sets. Since the arrangement of the cracks is that of an equi-distributed array, the irreversibility condition turns out to restrict the number $n-1$ of admissible cracks. The requirement for the admissible crack-set to contain all the previous ones implies that, after the first crack, the next possible crack set is the one with two added cracks in the middle of each segment, and so on. In summary, the admissible crack sets must be of the form of $n_{j}-1$ equally spaced cracks splitting the film in $n_{j}$ pieces of length $L / n_{j}$, with $n_{j}:=2^{j}, j \in\{0,1,2, \ldots\}, i . e$. the number of pieces has to be a power of two. Hence, the time-continuous quasi-static evolution of the crack-set of an initially sound film is found specializing the results of Proposition 1 to this case. In particular, the solution of the evolution problem is with $n_{j}-1$ equally spaced cracks for loadings in the interval $t^{\left(n_{j}-1\right)}<t<t^{\left(n_{j}\right)}$ with $t^{\left(n_{j}\right)}=t_{f}\left(L / n_{j}\right), t^{(0)}=0$, where

$$
t_{f}(L)=\frac{1}{\sqrt{2 \tanh \frac{L}{4}-\tanh \frac{L}{2}}}
$$

is the critical load for which a sound film of length $L$ is split in two equal parts by a transverse crack in the middle. The total energy of the solution obtained in this way and the number or segment $n$ versus the load $t$ are plotted in Figures 10(a) and 10(b), respectively. These figures emphasize how the irreversibility condition introduces a further selection among the admissible energy minimizers. 


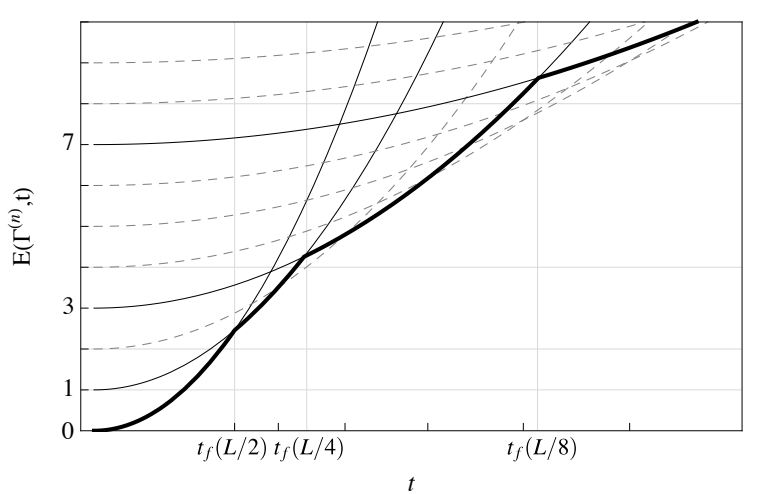

(a)

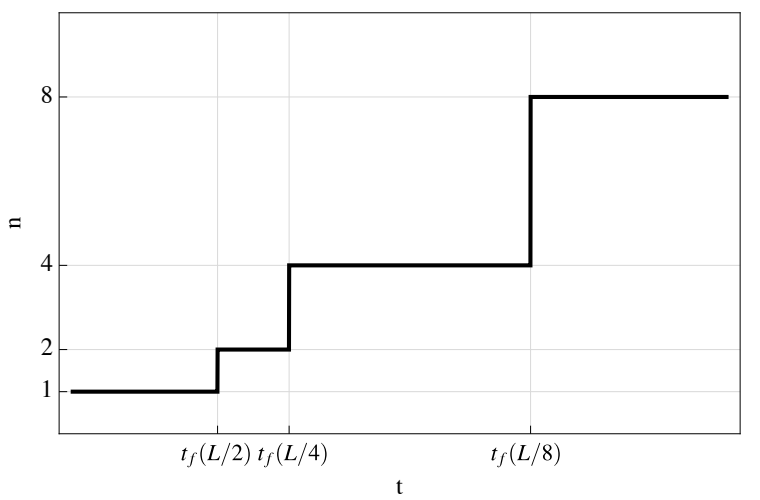

(b)

Fig. 10 Quasi-static evolution without debonding: (a) Energy curves $E\left(\Gamma^{(n)}, t\right)$ vs $t$ for different $n$, where the thick solid line denotes the global minimum corresponding to the solution of the evolution problem; (b) Number of parts $n_{j}$ of length $L / n_{j}$ vs $t$ fomed by the $n_{j}-1$ equally spaced cracks, with $n_{j}=2^{j}$.

\subsection{Perfectly elastic film subject to debonding only}

After the static solution of the debonding problem without cracks, its quasi-static counterpart is quite trivial. The irreversibility condition is a two-fold requirement: on one hand the size of the debonded domain must be non decreasing, on the other hand a stronger pointwise irreversibility must be ensured. This means that if at point $x$ the film is debonded at a time $t$, it has to be so for $\tau>t$. The first requirement is automatically satisfied by the static solution given in Proposition 2. Indeed the bonded length $B_{t}$ given in Equation (32) is strictly decresing with $t$. The second condition is fulfilled provided that the lengths of the debonded intervals $D_{1}(t)$ and $D_{2}(t)$, in the definition of the displacement field of Equation (28), are arbitrary non-decreasing functions of $t$. Hence, the result synthesized in Proposition 2 holds for the quasi-static evolution for the debonding problem replacing " $D_{1}$ and $D_{2}$ are arbitrary" with " $D_{1}(t)$ and $D_{2}(t)$ are arbitrary non-decreasing functions of $t$ ". In the case of a quasi-static evolution, these results may be interpreted saying that a bar of dimensionless length $L$ and relative cracking toughness $\gamma$, does not debond for $t<t_{b}(L, \gamma)$. At $t=t_{b}(L, \gamma)$ it starts debonding and the length of the (unique) bonded interval is $L_{b}(t, \gamma)$.

\subsection{Film subject to coupled transverse cracks and debonding}

The analysis of the static problem in the coupled case concludes with the Proposition of 3 and 4 , which are the starting point to construct solutions of the corresponding quasi-static problem. Irreversibility imposes that the number of transverse cracks are in the form $n_{j}=2^{j}-1$, with $j \in \mathbb{N}$ non-decreasing with $t$, and that the debonded domain is non-decreasing with $t$. For an imposed number $n-1$ of transverse cracks, the static solution given in Proposition 2 may be directly transposed to the quasi-static setting. Debonding starts appearing at the same time in each segment, at the critical load $t_{b}(L / n, \gamma)$ given by Equation (34). The energy of the solution is given by $\hat{E}_{t}^{(n)}$ of Equation $(38)$. The length of the bonded part in each piece is $L / n$ for $t<t_{b}(L / n, \gamma)$ and $L_{b}(t, \gamma) / n$ for $t>t_{b}(L / n, \gamma)$, with $L_{b}(t, \gamma)$ given by Equation (33). This solution respects the irreversibility because $L_{b}(t, \gamma)$ is non-increasing with $t$. Of course, the lengths of the debonded domains in each piece, $D_{1, i}$ and $D_{2, i}$, must be non-decreasing functions of $t$.

The determination of the evolution of the optimal number of transverse cracks is not an easy problem, in the general case. We illustrate here a typical quasi-static evolution for specific values of the film parameters $L$ and $\gamma$ when starting from a sound film at $t=0$. We trace for each admissible value of $n$ in the form $n_{j}=2^{j}$ the corresponding total energy as a function of the loading $t$, according to Equation (38) (gray lines in Figure 11(a). The evolution of the film satisfying the (GST) and (IR) conditions is the one corresponding to the lowest value of the energy and marked with a thick black stroke in Figure 11(a) This evolution satisfies also the energy balance (EB) because of the continuity of the energy and is therefore a well-defined solution of the quasi-static evolution problem. The corresponding displacement fields of the film are illustrated in Figure 12. Figure 11(b) reports the evolution of bonded length in each segment (thick black stroke). At $t=0$, it is 
equal to the total length of the sound bar. At the point $A$, which corresponds to the intersection with the curve $t_{f}(B)$ given by Equation (39) and marked with a thin solid gray stroke in Figure 11(b), the film breaks in $n=2$ parts without debonding. Then, the bonded length in each segment is $B=L / 2$, till the next intersection with the line $t_{f}(B)$ at point $C$, where each part further splits in two totally bonded segments of length $B=L / 4$. At point $D$ the load reaches the critical debonding time $t_{b}(B, \gamma)$ of Equation (34). Here, the film split into $n=4$ pieces starts debonding. No further transverse crack appears for higher loading.

More in general there may be a cascade of $\bar{j}$ transverse cracks followed by debonding. An interesting property of the solution is the number $n_{\bar{j}}$, which is the maximum number of pieces (of equal length) in which the film splits before delamination. As done in the static problem, this number is obtained by looking for the smallest integer value of $j$ for which $t_{b}\left(L / n_{j}, \gamma\right)<t_{f}\left(L / n_{j}\right)$. Figure 13 reports a phase diagram giving the $n_{j}$ obtained in this way as a function of the relevant parameters $L$ and $\gamma$.

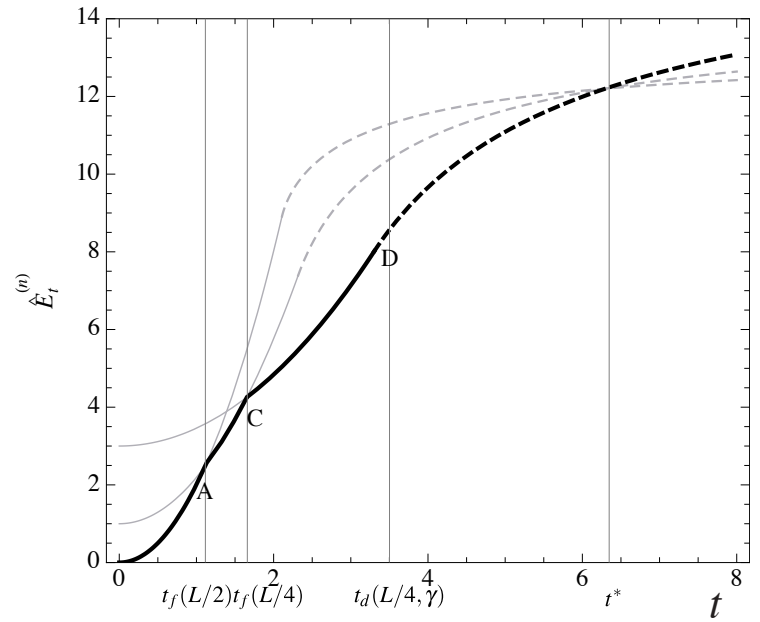

(a)

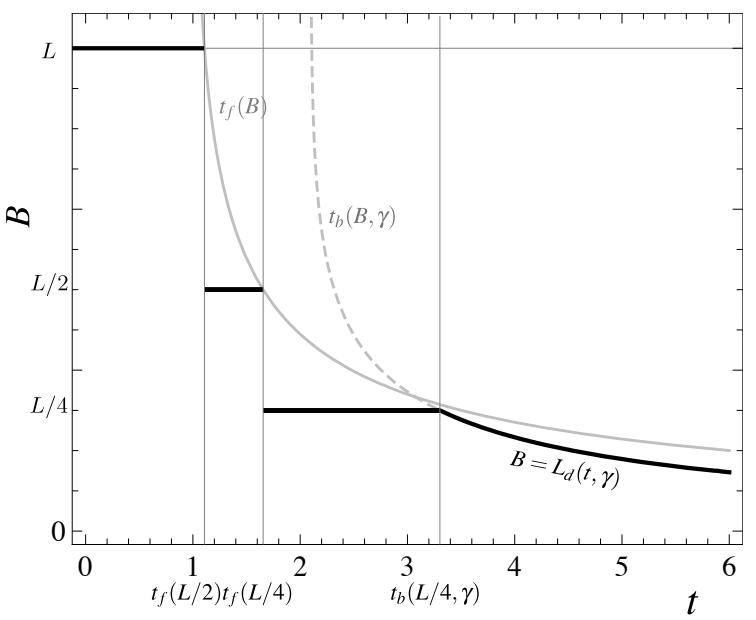

(b)

Fig. 11 Quasi-static evolution of an initially sound thin film of length $L=6$, with $\gamma=2.2$. (a) In gray, total energies calculated according to Equation (38) as a function of the load $t$ for different number of film pieces, the bonded states being marked by the dashed stroke. In black, the energy of the quasi-static evolution respecting (GST), (IR) and (EB). (b) In black, the length $B$ of the bonded interval in each film piece as a function of the load $t$. The continuous and dashed gray lines are the critical loads for transverse cracking and debonding of a sound film of length $B$ given by $t_{f}(B)$ and $t_{b}(B, \gamma)$, respectively.

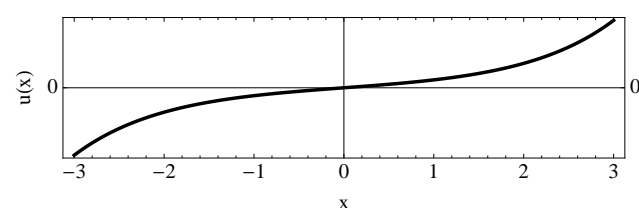

(a)

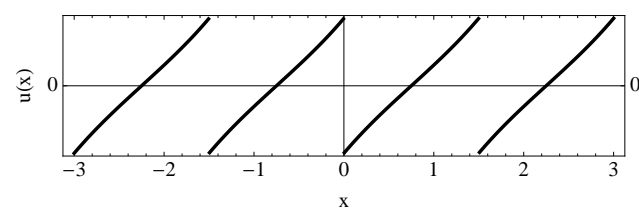

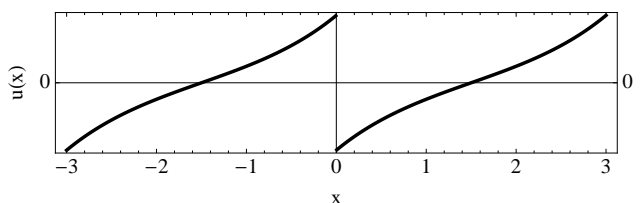

(b)

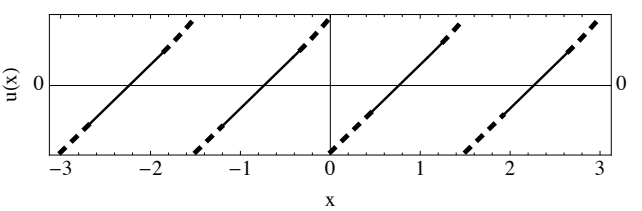

(c)

(d)

Fig. 12 Snapshots of the displacement field for states O, A, C, D of Figure 11(a) The debonded region is indicated with a thicker dashed line. 


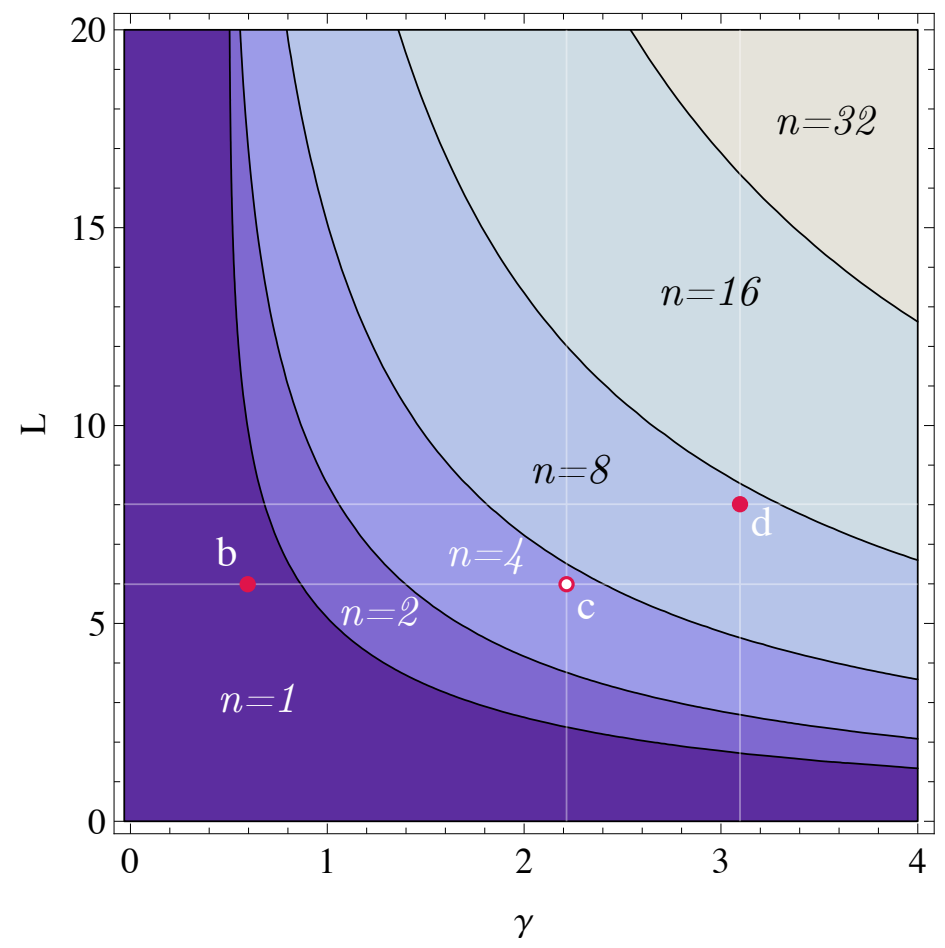

Fig. 13 Phase diagram for the coupled transverse cracking and debonding problem. For a quasi-static evolution, to each couple of parameters $(L, \gamma)$ the phase diagram associates the (maximum) number of parts $n$ into which the film is split before debonding takes place. We point out with a white dot the couple of parameters of the evolution illustrated in Section 5.3, with a red dot the couples of parameters referring to the numeric experiments detailed in Section 6

\section{Numerical experiments}

\subsection{Implementation}

The numerical approach we use is derived from the one presented in depth in [12,13]. It is based on a variational approximation of the total energy $\tilde{E}_{t}$ in (6) by elliptic functionals, originally proposed in [19,20] for the approximation of the Mumford-Shah problem in image processing. In the setting of [12,13], one can introduce a function $\alpha$ taking its values in $[0,1]$ and representing in some sense the crack set, a regularization parameter $\eta$ homogeneous to a length, and a regularized energy depending on the parameter $\eta$. It is then possible to show that this energy converge in the sense of $\Gamma$-convergence to the fracture total energy, from which one derives convergence of global minimizers (see [24] for instance for more details on the construction of the regularized energy). This analysis can then be carried out for the time evolution as shown in [25].

Here, we proceed by induction. We modify the regularized functional originally used in [12] to account for the inelastic strain and the debonding terms. For any $u, \alpha$, and any function $\delta$ with values in $\{0,1\}$ representing the debonded area, we consider a regularized energy in the form

$$
\tilde{\mathscr{E}}_{t, \eta}(u, \alpha, \delta):=\int_{\mathscr{A}} \frac{1}{2}(1-\alpha(x))^{2}\left(u^{\prime}(x)-t\right)^{2}+\frac{1}{2} u(x)^{2}(1-\delta(x))+\gamma \delta(x)+\frac{3}{8}\left(\frac{\alpha(x)}{\eta}+\eta \alpha^{\prime}(x)^{2}\right) d x .
$$

The surface energy term $\frac{3}{8} \int_{\mathscr{A}} \frac{\alpha}{\eta}+\eta \alpha^{\prime 2} d x$ differs from the one used in [12] while still falling with the more general scope of [24]. A comparison of the properties of both approximations can be found in [26].

We approach the time-continuous quasi-static evolution (CEL) by a time discretized regularized evolution law by considering a discrete set of loads $t_{i}$, and iteratively seeking global minimizers $\left(u_{i}, \alpha_{i}, \delta_{i}\right)$ of $\tilde{\mathscr{E}}_{t_{i}}, \eta$ under the crack growth condition $\alpha_{i} \geq \alpha_{i-1}$ and $\delta_{i} \geq \delta_{i-1}$. This time discrete minimization problem is in turn discretized in space by means of linear finite elements, following the lines of past work cited above. We made no attempt at proving the convergence of the regularized model or its dicretization to the continuous evolution law, a technical task beyond the scope of this article and under current investigation. Still, we expect that as 
$\eta \rightarrow 0$, the solution of the time discrete regularized model converge to that of the continuous time evolution, and in particular that the function $\alpha$ converges to 0 "along the cracks" and to 1 "away from the cracks", that the bulk term $\frac{1}{2} \int_{\mathscr{A}}(1-\alpha)^{2}\left(u^{\prime}-t\right)^{2} d x$ converge to $\frac{1}{2} \int_{\mathscr{A} \backslash \Gamma}\left(u^{\prime}-t\right)^{2} d x$, and that $\frac{3}{8} \int_{\mathscr{A}}\left(\frac{\alpha}{\eta}+\eta \alpha^{\prime 2}\right) d x$ converges to the number of cracks in the domain.

The numerical minimization of the regularized energy is challenging as it is non-convex and stiff. For each loading parameter $t_{i}$, we take advantage of the fact that $\tilde{\mathscr{E}}_{t, \eta}$ is separately convex in the variables $u, \alpha$ and $\delta$ and alternate minimization with respect to $u, \delta$, and $\alpha$ until convergence. Minimization with respect to the displacement field $u$ is a straightforward unconstrained optimization problem implemented using a preconditioned conjugated gradient algorithm. Following Lemma 11, we know that for any $t_{i}$, the optimal $\delta_{i}$ is a characteristic function (i.e. no relaxation phenomenon takes place), and moreover that for a given $u$ and $\alpha$ the optimal function $\delta(x)$ is such that $\delta(x)=1$ if $u(x) \geq \sqrt{2 \gamma}$ and 0 otherwise. Finally, minimization with respect to $\alpha$ is a box constrained problem.

It is well known that such an algorithm applied to a non-convex energy may not converge to a global minimizer, but only to a critical point. In the case of the uncoupled problems (transverse fracture or debonding only), this can me mitigated by implementing a backtracking algorithm, relying on a necessary condition for optimality with respect to the time evolution, and a construction of an evolution which satisfies it [27]. In the current situation, when the competition takes place between three terms in the energy, a similar optimality condition can be written, but the construction of an evolution satisfying it is not as straightforward, and the results presented in the sequel do not use a backtracking algorithm. Note however that the properties in Lemma 1 and 2 about the general properties of solutions with debonding only require that first order optimality conditions be satisfied and therefore hold for local minimizers and critical points of the total energy. Finally, note that there is always a debate surrounding global minimality as the proper framework. In short global minimality may sometimes lead to unphysical evolutions, but local minimality typically forbids transverse crack initiation without singularity, the essence of the one-dimensional model. We do not take part in this debate, but notice that in most cases, in our experiments, transitions between energy-minimizing states are driven by the loss of their stability, which in turn is sensitive to the parameter $\eta$.

We conclude the description of the numerical approach by two remarks. First, as we focus here on the simple one-dimensional situation, our numerical experiments do not illustrate one of the strengths of our approach, its ability to handle complicated crack geometries and topologies. In the one-dimensional setting, there are methods based on dynamic programming that are guaranteed to converge to global minimizers of the total energy. Of course, this is at the cost of versatility as these do not generalize easily to the multidimensional case. In contrast, our approach is suited to the numerical simulation of real two-dimensional problems, in situations where complicated crack and debonding geometries are expected, a strength that is not fully exploited in the present work. Secondly, the regularized energy 40 is similar to a non-local damage model where $\alpha$ is a damage parameter and $\eta$ an internal length. In the current study however, we see this as a mere coincidence. We only think of of $\eta$ as a numerical regularization parameter and of $\tilde{\mathscr{E}}_{t, \eta}(u, \alpha, \Delta)$ as an approximation of (6), taking place as $\eta \rightarrow 0$.

We propose to use this numerical approach to illustrate the various results presented in the previous sections, starting with quasi-static transverse fracture and debonding separately, then coupling both fracture modes. In addition to the scaling (7), we modify the toughness $\gamma$ in order to account for the interaction between the mesh discretization and regularization parameters as in [13].

For each experiment we present, we use the following convention: in the energy plots, the total energy is plotted in bold solid line, the energy associated with the transverse cracks in dashed lines, that associated with the debonding in dot-dashed lines, and the elastic energy in thin solid lines. When applicable, the total energy of the closed form solution is also plotted in light dashed lines, and the relevant critical loads indicated by vertical dashed lines. When we plot the displacement and the $\alpha$ field representing the cracks, the displacement is depicted in thick solid lines and $\alpha$ as a thin line. When we plot the displacement and the debonding characteristic function $\delta$, the displacement is depicted in thick solid lines and $\delta$ as a thin line. The displacements are normalized so that they take values in $(-1,1)$. The debonded area corresponding to $\delta(x)=1$ is highlighted in light gray.

\subsection{Numerical simulation of a perfectly bonded film subject to transverse cracks only}

We focus first on the case of a perfectly bonded film studied in Section 4.2 where only transverse cracks are allowed. This is achieved by forcing $\delta \equiv 0$ in our numerical scheme. We consider a domain of length $L=6$, 
decomposed into 600 linear finite elements $\left(h=10^{-2}\right)$. The load history $\left[O, T_{\max }\right]$ is discretized with 600 time steps of equal length. The regularization parameter $\eta$ is set to $5.510^{-2}$.

Figure 14 presents the outcome this experiment. The snapshot of the displacement and damage fields in Figure 14(b) is taken at the last time step of the load path. The equi-distributed fracture pattern is correctly captured, indeed the peaks of the damage field representing the location of cracks occur at $x=-1.5,0,1.5$ in Figure 14(b). Moreover, the damage field has continuous derivative except at its peaks and decays rapidly to 0 . In the energy plot, the first branch of parabola corresponds to the elastic loading of the sound film. At $t=1.78$ the first transverse fracture appears at the center of the film, which leads to a sudden increase of the surface energy at the expense of elastic energy. As the loading increases, the system follows the second branch of parabola, $i$. $e$. the elastic loading of the $t w o$ pieces into which the original film is split. The second critical load $t=2.60$ corresponds the opening of two cracks along the center of each film piece. The final snapshot of the fracture profile is shown in Figure 14(b) As hinted above, the mechanisms leading to bifurcation between branches of energy is loss of stability and is $\eta$-dependent leading us to overestimate the value of the critical loads. In this situation, a backtracking algorithm would have allowed the reconstruction of the proper energy profile. We also note that in this situation, the displacement field is not perfectly antisymmetric with respect to the center of each of film pieces as expected. This is due to the fact that whereas the free boundary condition is exactly satisfied at the end point of the domain, it is only asymptoteically satisfied as $\eta \rightarrow 0$ along the crack. Indeed, in Figure 14(b), one can see that the the displacement magnitude is slightly at the boundary of the domain that at the cracks edges. As we will see below, this will have an effect on the debonding evolution in coupled simulations.

\subsection{Numerical simulation of a perfectly elastic film subject to debonding only}

We continue our numerical investigations by focussing on the situation considered in Section 5.2 where only debonding is allowed. Again, this is easily achieved by skipping the minimization with respect to $\alpha$ and forcing $\alpha \equiv 0$ throughout the domain. The discretization parameters are identical to that above, and the parameter $\gamma$ arbitrarily set to 0.5 . Figure 15 presents the outcome of our numerical experiments. The fields $u$ and $\delta$ at the last time step are shown in Figure 15(b). The area where $\delta(x)=1$ is highlighted in gray. As predicted in Proposition 2, the debonded area consists in two segments originating from each end of the domain. Although the analytic solution is not unique, our numerical solution seem to favor a symmetric solution. On the debonded domain, the displacement is linear and the deformation gradient is identically equal to the imposed inelastic strain.

The energy plot is shown in Figure 15(a) The critical load of 1.01 at which debonding takes place in the closed form solution in (33) is denoted by a vertical thin line. The critical load we obtained in the numerical experiment is 1.04, a relative error of 3\%. The comparison of the total size of the debonded domain yields $B=5.66$ using Equation (32), and $B_{h}=5.67$ for the numerical experiment, a relative error of $0.1 \%$. The surface energy related to debonding increases as $L-L_{b}(t)$. The total energy grows asymptotically to reach a value of $\gamma L$ of a fully debonded film.

The obtained evolution matches perfectly that obtained in Section 5.2 This is not really a surprised as the solution is given by the first order optimality conditions (31) which are implemented in the minimization algorithm.

\subsection{Numerical simulation of a film subject to coupled transverse cracks and debonding}

We finally present two numerical experiments implementing the fully coupled model. In the first one, the geometry and spacial discretization parameters are unchanged. The load increment is set to $10^{-2}$ and the parameter $\gamma$ to 2.2, matching the example from Figure 11 in Section 5.3 .

In the numerical experiment, we observe the nucleation of a transverse crack at the center for a load $t=1.59$, instead of the predicted $t=1.04$. Again, this is due to the fact that in the numerics, the bifurcation from the un-cracked configuration to the cracked one is governed by loss stability of the elastic solution, and not global minimality. For similar reasons, we do not observe the initiation of cracks on each of the ligaments as predicted in Section 5.3 . However, as expected, debonding expanding from the crack tip occurs at a load of $t=1.98$ (an error of .2\% compared to the theoretical value), and the evolution of the total debonded length follows the one predicted in Figure 11. In particular, the size of the debonded domain at the end of the loading 


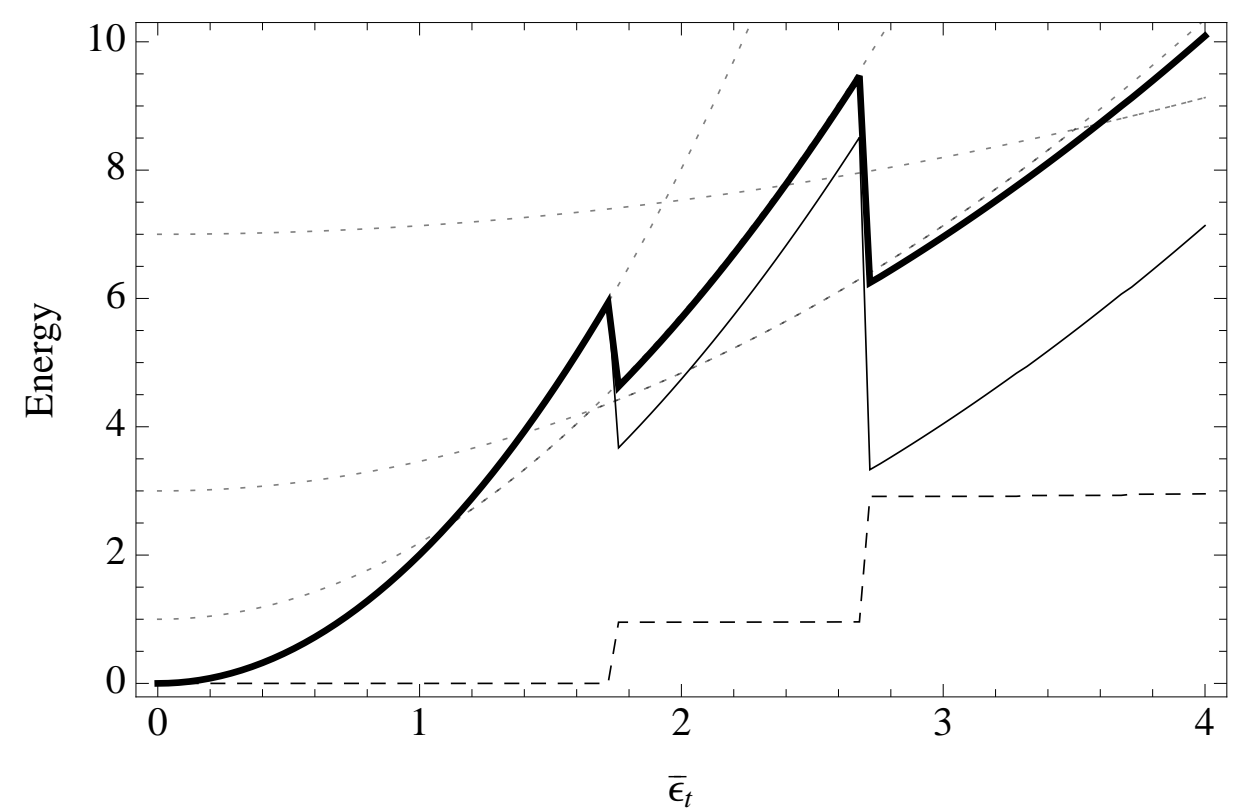

(a) Evolution of the system total (thick line), elastic (thin line) and surface energies (dashed). The total energy of the crack states studied in Section 4.2 is shown in light dashed curves.

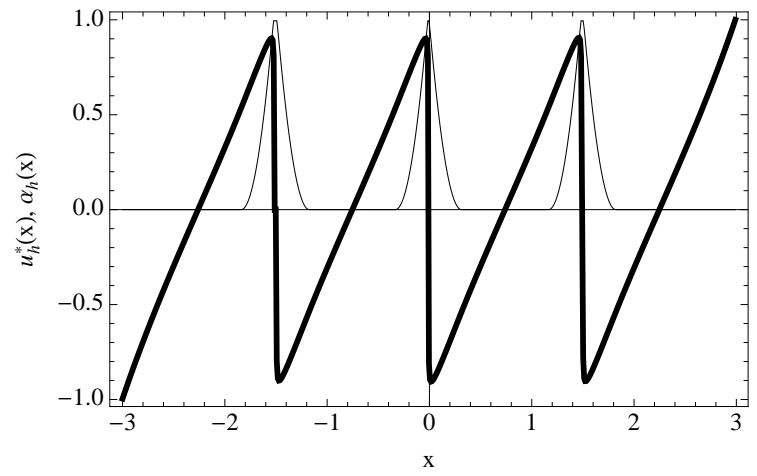

(b) At the end of the loading phase three transverse cracks are obtained, represented by the localization teh field $\alpha$ on a narrow zone. In our regularized formulation, discontinuities of the displacement are replaced with zones with very high gradient.

Fig. 14 Uncoupled numerical experiment allowing only transverse cracks.

phase is 7.34, matching the predicted value within $3 \%$. The value of the first critical load could be improved using a backtracking argument. However, since we do not observe nucleation of secondary cracks, but instead directly detect debonding, a simple backtracking argument will not suffice to identify the missed bifurcation.

In order to qualitatively recover the richness of the evolution described in Section 5.3 , we consider a second numerical simulation of a domain of length 8 , discretized into elements of size $1.610^{-2}$.

In this situation, we first obtain a centered transverse crack a $t=1.56$, followed by the simultaneous initiation of two cracks, centered around each piece of film at a critical load $t=2.04$. Only at this point is debonding of the film taking place. As hinted in Section 6.2, the displacement field is not perfectly antisymmetric in the first and last ligament, causing debonding to initiate there slightly earlier that at the film's cracks. This can be seen in Figure 17(c) where the debonded region is not symmetric in the outer most film pieces $(-4,-2)$ and $(2,4)$. The energy evolution we recover matches the prediction with a bonded area decreasing as $L_{b}(t)$ and a debonding energy proportional to $L-L_{b}(t, \gamma)$ as in Equation 33 The numerical critical debonding load is 3.12 it underestimates the critical load for debonding $t_{b}(L / 4)=3.24$ computed with Equation (34). 


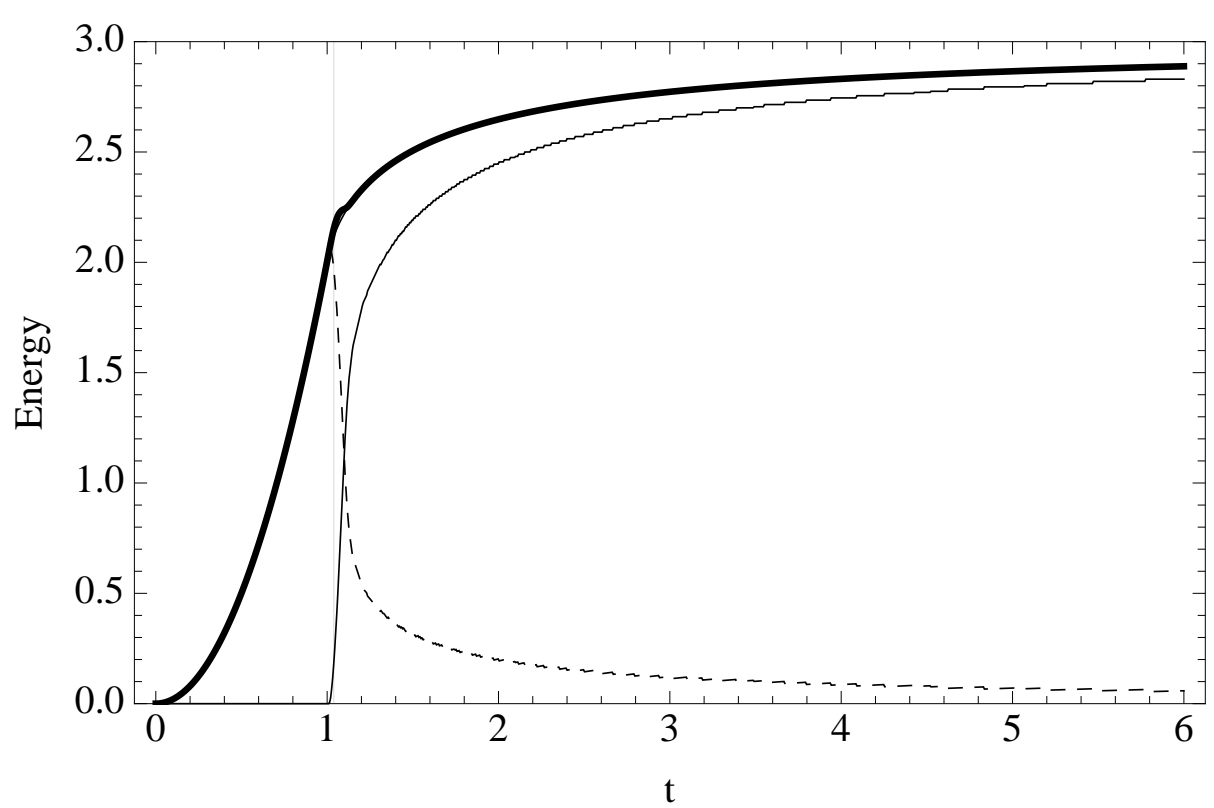

(a) Energy plot for the uncoupled debonding experiment. After an elastic loading phase, the film undergoes debonding. The total energy has an horizontal asymptote that corresponds to the energy of the totally debonded film.

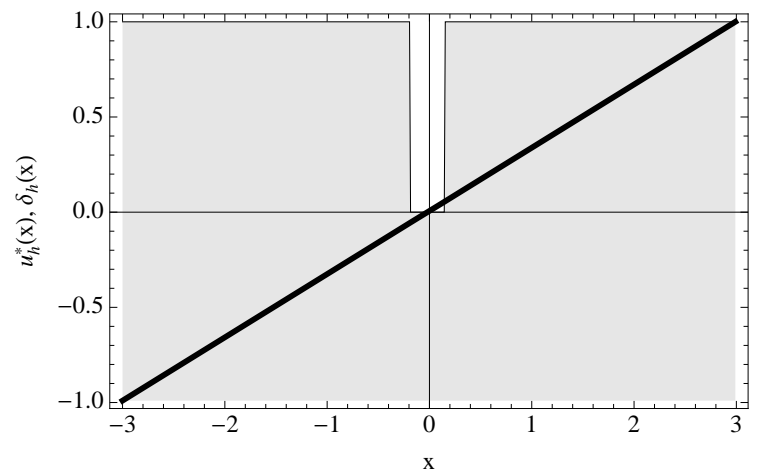

(b) Plot of the displacement field and the debonded domain (shown shaded in light gray) shaded field. As expected, debonding initiates at the edge of the domain and propagates towards its center. The displacement is linear on the debonded domain.

Fig. 15 Uncoupled numerical experiment allowing only debonding. 


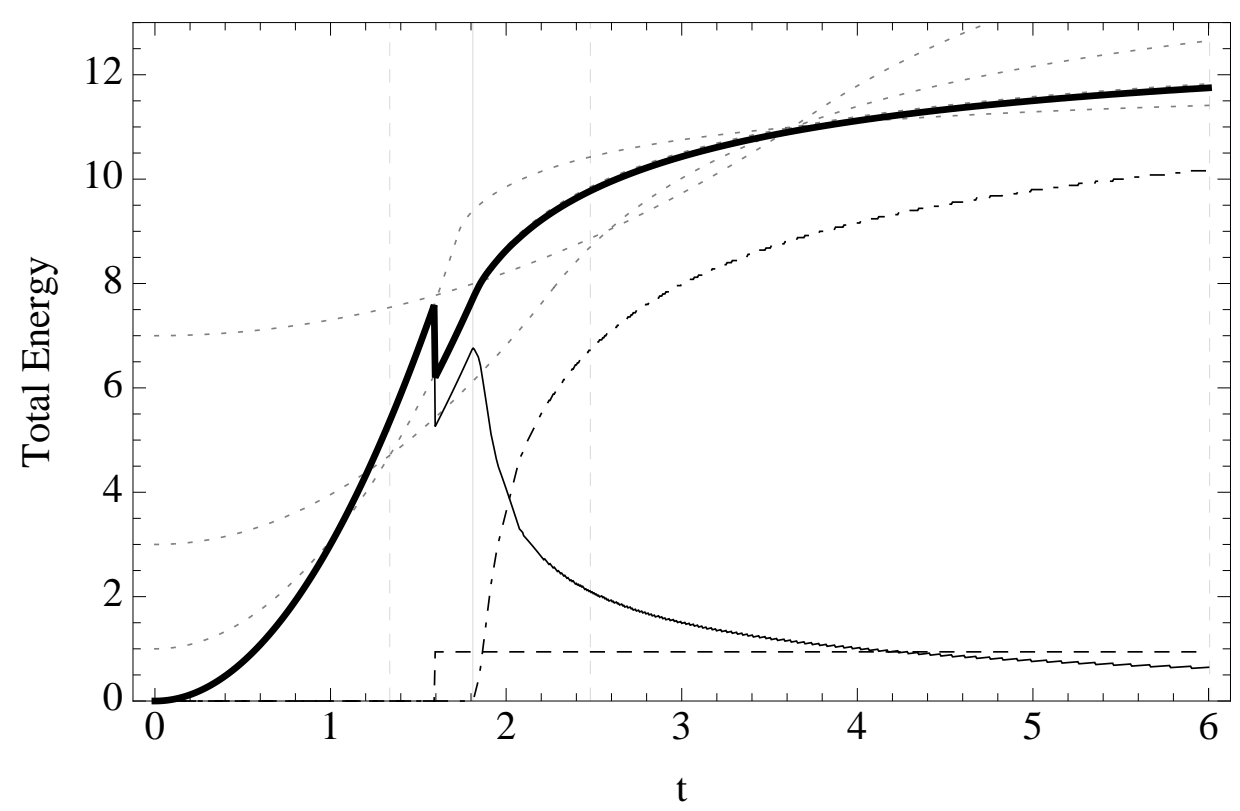

(a) Energy plot of the evolution, after a sound loading phase, the system jumps to the fractured state with one crack. The energy is discontinuous at the transition load. Then debonding starts respecting energy continuity.

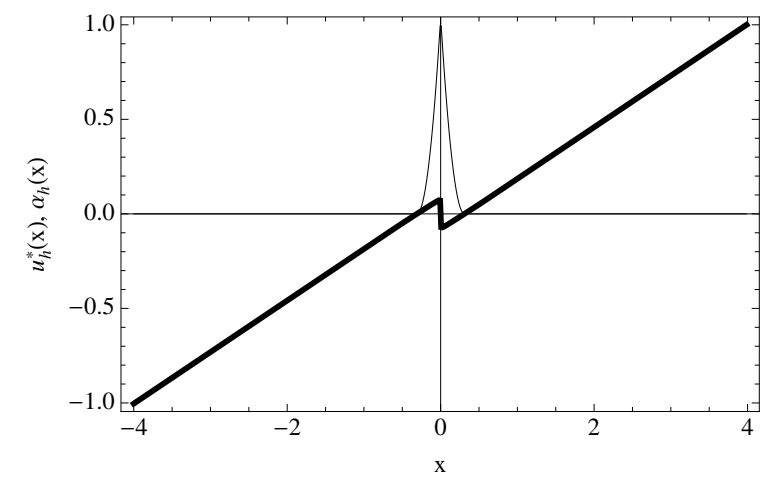

(b) Plot of the displacement and damage field. The crack appears in the middle of the film.

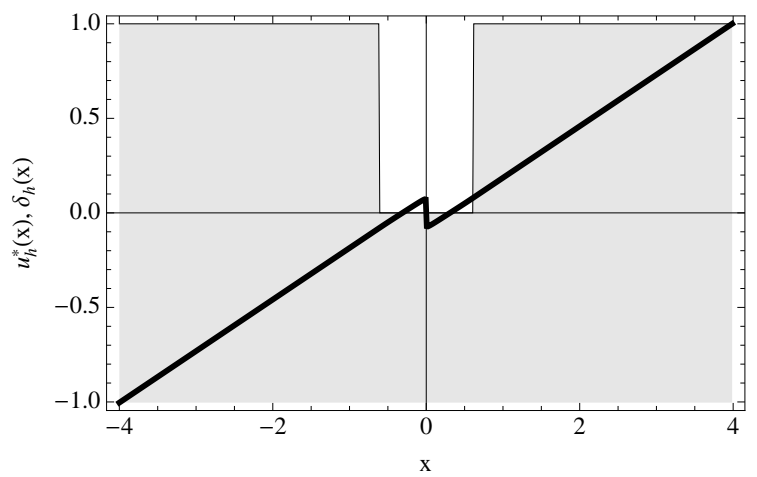

(c) Plot of the displacement and the debonded domain. It is symmetric with respect to the center of the film.

Fig. 16 Coupled experiment \#1: transverse fracture and debonding. One transverse fracture and then delamination. 


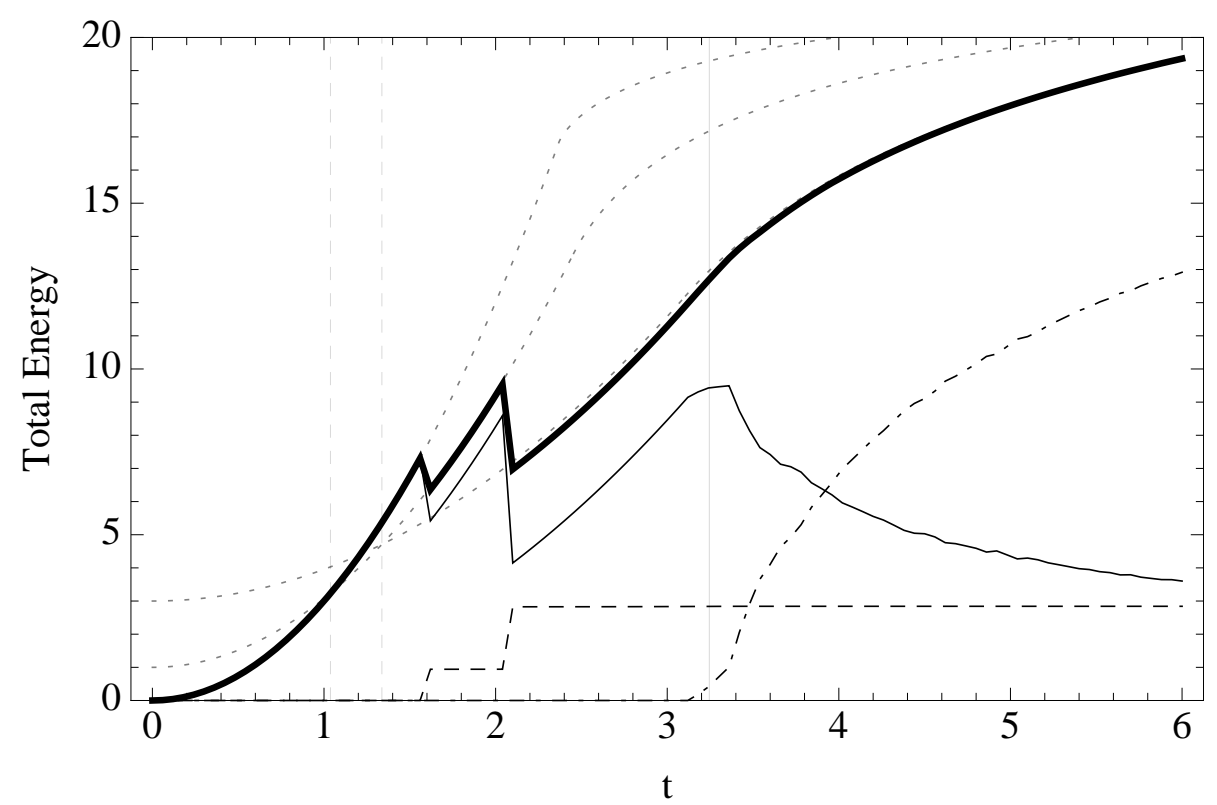

(a) Energy plot shows an evolution consisting in three subsequent transverse fractures and debonding. Boundary layer effects appear during the very first phase of debonding, as the linear growth of the debonding energy in the very first debonding phase indicates.
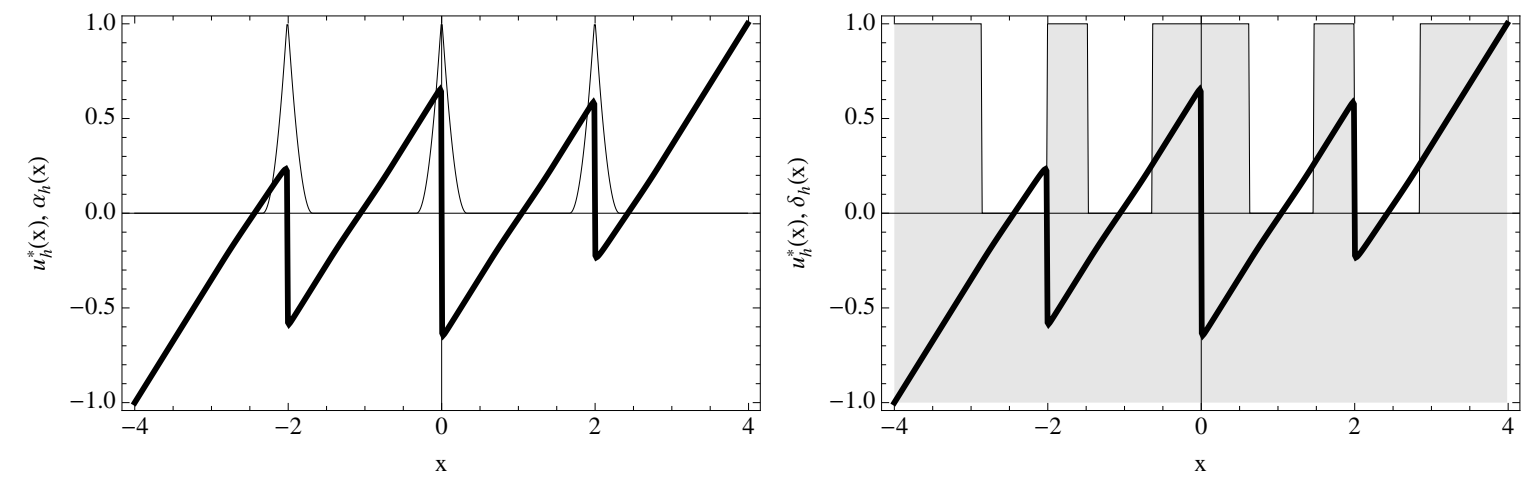

(b) Plot of the displacement and damage fields, the film ex- (c) Plot of the displacement and debonded domain (shaded in hibits three transverse cracks at the end of the loading phase. light gray). The latter is not symmetric for each part of the bar since the total energy is insensitive to the arrangement of the debonded domain.

Fig. 17 Coupled experiment \#2: transverse fracture and debonding. Three transverse fractures and debonding. 


\section{Conclusions}

We applied the variational approach to fracture mechanics to study fracture and debonding of a thin film bonded on a stiff substrate. Our analysis is based on a $1 D$ reduced model where transverse cracks and debonding are introduced through a surface energy of the Griffith type, the bulk energy being assumed to be linear elastic up to failure. The presence of the substrate is accounted for by an equivalent elastic foundation. We made no attempt here to present a rigorous justification of the model starting from the $3 D$ description, but instead we performed a detailed analysis of its solutions and their qualitative properties.

In the reduced model transverse and debonding cracks appears with a different geometric nature. Whereas the first are of geometrical co-dimension 1 (points in $1 D$ and surfaces in $2 D$ ), the second are of co-dimension 0 (segments in $1 D$ and surfaces in $2 D$ ). Only transverse cracks introduce discontinuities in the displacement fields. This difference entails a distinct mathematical and numerical treatment and solutions with disparate qualitative properties. This point has been emphasized by presenting separately the solutions of the transverse fracture and debonding problems, before tackling the more complex coupled case. For debonding without transverse cracks, the main result is that the bonded part of the domain is a single connected segment, which is uniquely determined as a function of the loading. Equivalently, debonding may appear only at the ends of the domain. Moreover, this is a property is true of all local minima of the energy.

The modeling of transverse cracks requires us to formulate problem in terms of global minimization, as customary in the variational approach to fracture mechanics with a Griffith-type surface energy. We showed that transverse cracks are equally spaced and lead to periodic solutions. This behavior was only postulated in previous studies. The coupling of transverse fracture and debonding produces an interesting and rich behavior even in the $1 D$ setting. Through analytical results and phase diagrams, we unveiled the dependence of the key qualitative properties of the solutions on the two non-dimensional parameters of the model. In the numerical part, we proposed a finite elements implementation of a regularized model. Our numerical approach is used to illustrate key properties of the model identified in the analysis section.

The present work is intended to constitute a solid background to treat more multiple cracks problems within the variational approach to fracture mechanics. A natural extension of this work is to tackle the $2 D$ case, which is known to lead to intriguing complex crack and debonding patterns, like fracture networks, parallel crack arrays with stop and go phenomena, or spirals. The extension of the numerical model to $2 D$ is straightforward. It is under active development and the analytical results presented here will be used for its verification. The rigorous derivation of the thin film model with fracture and debonding, starting from a $3 D$ variational model with Griffith surface energy is pending. Preliminary $\Gamma$-convergence results are available and will be reported in a forthcoming paper. Our final aim is to compare the analytical and numerical findings to experimental evidences. Collaborations with experimentalists in the thin film domain are currently in progress. 


\section{A Appendix}

Proof (of Lemma 1 ) We consider a sound bar $\Gamma=\emptyset$ and let $u$ be a local minimizer of $\tilde{\mathscr{E}}_{t}(u, \emptyset$ ).

Let us first prove that there exists $x \in[-L / 2, L / 2]$ such that $|u(x)|<u_{c}$, by contradiction. Let us consider the following family of admissible displacement fields $v_{h}=u+h v$ with $h>0$ and $v \in \mathscr{C}(\emptyset)$. Since $v_{h}$ converges to $u$ as $h \rightarrow 0$, we must have for $h$ sufficiently small

$$
0 \geq \tilde{\mathscr{E}}_{t}(u, \emptyset)-\tilde{\mathscr{E}}_{t}\left(v_{h}, \emptyset\right)=\int_{-L / 2}^{L / 2}\left(2 h\left(u^{\prime}-t\right) v^{\prime}+h^{2} v^{\prime 2}+f(|u(x)|)-f\left(\left|v_{h}(x)\right|\right)\right) d x
$$

Since $f(|u(x)|)=\gamma \geq f\left(\left|v_{h}(x)\right|\right)$, one gets $0 \geq \int_{-L / 2}^{L / 2}\left(2 h\left(u^{\prime}-t\right) v^{\prime}+h^{2} v^{\prime 2}\right) d x$. Dividing by $h$ and passing to the limit as $h \rightarrow 0$, one obtains $\int_{-L / 2}^{L / 2}\left(u^{\prime}-t\right) v^{\prime} d x=0$, equality which must hold for every $v \in \mathscr{C}(\emptyset)$. It is possible only if $u^{\prime}=t$. Inserting this relation into (41) leads to a contradiction. Therefore there exists $x \in[-L / 2, L / 2]$ such that $|u(x)|<u_{c}$.

Then, let us prove that $u$ has (at least) a zero in $[-L / 2, L / 2]$, still by contradiction. Suppose that $u$ has the same sign on $[-L / 2, L / 2]$, say $\varepsilon= \pm 1$. For $h \in(0, \min |u|)$, let us consider the following family of admissible displacement fields $v_{h}$ :

$$
v_{h}(x)=u(x)-\varepsilon h .
$$

Since $v_{h}$ converges to $u$ as $h \rightarrow 0$, we must have for $h$ sufficiently small

$$
0 \geq \tilde{\mathscr{E}}_{t}(u, \emptyset)-\tilde{\mathscr{E}}_{t}\left(v_{h}, \emptyset\right)=\int_{[0, L]} f(|u(x)|)-f\left(\left|v_{h}(x)\right|\right)
$$

But since $\left|v_{h}(x)\right|<|u(x)|$ and since $f$ is strictly increasing in the interval $\left[0, u_{c}\right]$, the above inequality can be satisfied only if $|u(x)| \geq u_{c}$ for all $x$. But we have proved before that it is impossible. Therefore $u$ has at least a zero.

We are now in a position to prove that $u$ is monotonic, by contradiction. If $u(x)$ is not monotonic, then it has a positive local maximum or a negative local minimum in $(-L / 2, L / 2)$ and we can find $U \in \mathbb{R}$ such that there exist $-L / 2 \leq a<b \leq L / 2$ verifying:

$$
u(a)=u(b)=U \quad \text { and } \quad|u(x)|>|U|, \quad \forall x \in(a, b)
$$

For $h \in(0,1)$, let us consider the following family of admissible displacement fields $v_{h}$ :

$$
v_{h}:= \begin{cases}u(x), & \text { if } x \notin(a, b) \\ (1-h) u(x)+h U & \text { if } x \in[a, b]\end{cases}
$$

Since $v_{h}$ converges to $u$ as $h \rightarrow 0$, we must have for $h$ sufficiently small

$$
\begin{aligned}
0 & \geq \tilde{\mathscr{E}}_{t}(u, \emptyset)-\tilde{\mathscr{E}}_{t}\left(v_{h}, \emptyset\right) \\
& =\int_{[a, b]}\left(u^{\prime}(x)-t\right)^{2}+f(|u(x)|) d x-\int_{[a, b]}\left(u^{\prime}(x)(1-h)-t\right)^{2}-f\left(\left|v_{h}(x)\right|\right) d x \\
& =\int_{[a, b]} u^{\prime 2}(x)\left(1-(1-h)^{2}\right) d x-2 h \int_{[a, b]} t u^{\prime}(x) d x+\int_{[a, b]} f(|u(x)|)-f\left(\left|v_{h}(x)\right|\right) d x \\
& =\int_{[a, b]} u^{\prime 2}(x)\left(1-(1-h)^{2}\right) d x+\int_{[a, b]} f(|u(x)|)-f\left(\left|v_{h}(x)\right|\right) d x
\end{aligned}
$$

Since both the integrands are non negative, for the inequation to be satisfied both the integrands must vanish. This leads to $u^{\prime}(x)=0$ in $(a, b)$ and since $u(x)=U$ for $x=a, b$ and $u$ must be continuous, one should have $u(x)=U$ in $(a, b)$ which contradicts $|u(x)|>|U|$ in $(a, b)$. Then $u(x)$ is monotonic.

Proof (of Lemma 2) Let $v \in C_{0}^{\infty}([-L / 2, L / 2])$ and $h \in \mathbb{R}$. When $|h|$ is sufficiently small, then $\varphi_{h}(x):=x+h v(x)$ is a $C^{\infty}$ diffeomorphism on $[-L / 2, L / 2]$. Let $u \in W^{1,2}[-L / 2, L / 2]$, and define $u_{h}:=u \circ \varphi_{h}^{-1}$. One has $\lim _{h \rightarrow 0} u_{h}(x)=u(x)$ pointwise. If $u$ is a local minimizer then $I(u) \leq I\left(u_{h}\right)$ for some sufficiently small $h$. We compute

$$
I\left(u_{h}\right)=\int_{0}^{L}\left(\left(\frac{u^{\prime}(y)}{\varphi_{h}^{\prime}(y)}-t\right)^{2}+f(|u(y)|)\right) \varphi_{h}^{\prime}(y) d y
$$

The last quantity is differentiable with respect to $h$, attaining its minimum value for $h=0, u$ being a minimizer. We therefore require that the first derivative with respect to $h$ vanishes for $h=0$

$$
0=\left.\frac{d I\left(u_{h}\right)}{d h}\right|_{h=0}=\int_{0}^{L}\left(-\left(u^{\prime 2}(x)-t^{2}\right)+f(|u(x)|)\right) v^{\prime}(x) d x, \quad \forall v \in C_{0}^{\infty}([-L / 2, L / 2])
$$

We easily infer the prime integral: $u^{\prime}(x)^{2}-t^{2}-f(|u(x)|)=C, \forall x \in[-L / 2, L / 2]$. Let's compute the boundary conditions. Define $x_{h}=L / 2(1-h)$ for $h \in(0,1)$ and $\forall \vartheta \in \mathbb{R}$ construct a test field $v_{h}$ as follows

$$
v_{h}(x):= \begin{cases}u(x), & \text { if } x \in\left[-L / 2, x_{h}\right] \\ u(x)+\vartheta\left(x-x_{h}\right) & \text { if } x \in\left[x_{h}, L / 2\right]\end{cases}
$$


Such a test field is admissible and $v_{h} \rightarrow u$ pointwise for $h \rightarrow 0$. Let $u$ be a minimizer, then we can write the following inequality

$$
\begin{aligned}
0 & \leq \tilde{\mathscr{E}}_{t}\left(v_{h}(x), \emptyset\right)-\tilde{\mathscr{E}}_{t}(u, \emptyset) \\
& =\int_{\left[x_{h}, L\right]}\left(u^{\prime}(x)-t\right)^{2}+f(|u(x)|) d x-\int_{\left[x_{h}, L\right]}\left(u^{\prime}(x)+\vartheta-t\right)^{2}-f\left(\left|v_{h}(x)\right|\right) d x \\
& =\int_{\left[x_{h}, L\right]} \vartheta^{2}+2 \vartheta u^{\prime}(x)-2 \vartheta t+\left(f\left(\left|v_{h}(x)\right|\right)-f(|u(x)|)\right) d x
\end{aligned}
$$

dividing the last equation by $h$ and passing to the limit for $h \rightarrow 0$, the term in parentheses vanishes grace to the pointwise convergence $v_{h} \rightarrow u$ and the inequality

$$
0 \leq \vartheta^{2}+2 \vartheta\left(u^{\prime}(L)-t\right)
$$

has to be verified $\forall \vartheta \in \mathbb{R}$. This leads to the desired boundary condition: $u^{\prime}(L / 2)=t$. Symmetrically we can construct test fields to retrieve the boundary condition on $x=0$ as follows

$$
v_{h}(x):= \begin{cases}u(x)+\vartheta\left(x-x_{h}\right) & \text { if } x \in\left[-L / 2, x_{h}\right] \\ u(x), & \text { if } x \in\left[x_{h}, L / 2\right]\end{cases}
$$

where now $x_{h}=h$. We have the same pointwise convergence as above and we derive the boundary condition $u^{\prime}(-L / 2)=t$.

\section{Acknowledgements}

This joint work was financed by the French National Research Council CNRS in the framework of a joint international research program PICS. B. Bourdin's work was supported in part by the National Science Foundation under the grant DMS-0909267. J.-J. Marigo and C. Maurini gratefully acknowledge the funding of the ANR program T-Shock OTP J11R087. C. Maurini and A.A.Leon Baldelli work was partially supported by University Pierre et Marie Curie in the framework of an EMERGENCESUPMC grant.

\section{References}

1. Hutchinson, J.W., Suo, Z.: Mixed mode cracking in layered materials. Advances in Applied Mechanics 29 (1992)

2. Timm, D.: Prediction of thermal crack spacing. International Journal of Solids and Structures 40(1), 125-142 (2003)

3. Lazarus, V., Pauchard, L.: From craquelures to spiral crack patterns: influence of layer thickness on the crack patterns induced by desiccation. Soft Matter 7(6), 2552 (2011)

4. Hsueh, C.H.: Analyses of multiple film cracking in film/substrate systems. Ceramics 84(12), 2955-2961 (2001)

5. Zhang, T.y., Zhao, M.h.: Equilibrium depth and spacing of cracks in a tensile residual stressed thin film deposited on a brittle substrate. Engineering Fracture Mechanics 69, 589-596 (2002)

6. Bialas, M., Mroz, Z.: Crack patterns in thin layers under temperature loading. Part I: Monotonic loading. Engineering Fracture Mechanics 73(7), 917-938 (2006)

7. Bialas, M., Mroz, Z.: An energy model of segmentation cracking of thin films. Mechanics of Materials 39(9), 845-864 (2007)

8. Hsueh, C.H., Yanaka, M.: Multiple film cracking in film / substrate systems with residual stresses and unidirectional loading Journal of Materials Science 38, 1809 - 1817 (2003)

9. Kim, S.: Fracture mechanics analysis of coating/substrate systems Part I: Analysis of tensile and bending experiments. Engineering Fracture Mechanics 65(5), 573-593 (2000)

10. Xia, Z.C., Hutchinson, J.W.: Crack patterns in thin films. Journal of the Mechanics and Physics of Solids 48, 1107-1131 (2000)

11. Francfort, G.A., Marigo, J.J.: Revisiting Brittle Fracture as an Energy Minimization Problem. Journal of the Mechanics and Physics of Solids 46, 1319-1342 (1998)

12. Bourdin, B., Francfort, G., Marigo, J.J.: Numerical experiments in revisited brittle fracture. Journal of the Mechanics and Physics of Solids 48(4), 797-826 (2000)

13. Bourdin, B., Francfort, G., Marigo, J.J.: The variational approach to fracture. Journal of Elasticity 91(1-3), 1-148 (2008)

14. Del Piero, G., Owen, D.R.: Structured deformations of continua. Archive for Rational Mechanics and Analysis 124, $99-155$ (1993)

15. Del Piero, G., Truskinovsky, L.: Macro- and micro-cracking in one-dimensional elasticity. International Journal of Solids and Structures 38, 1135-1148 (2001)

16. Del Piero, G., Truskinovsky, L.: Elastic bars with cohesive energy. Continuum Mechanics and Thermodynamics 21(2), 141-171 (2009)

17. Lancioni, G., Royer-Carfagni, G.: The variational approach to fracture mechanics. A practical application to the French Panthéon in Paris. Journal of Elasticity 95, 1-30 (2009)

18. Mielke, A.: Evolution of rate-independent systems. In: Evolutionary equations, Handb. Differ. Equ., vol. II, pp. 461-559. Elsevier/North-Holland, Amsterdam (2005)

19. Ambrosio, L., Tortorelli, V.M.: Approximation of functional depending on jumps by elliptic functional via $\Gamma$-convergence. Communications on Pure and Applied Mathematics 43(8), 999-1036 (1990) 
20. Ambrosio, L., Tortorelli, V.: On the approximation of free discontinuity problems. Bollettino della Unione Matematica Italiana. Serie VIII. Sezione B. 6(1), 105-123 (1992)

21. Nguyen, Q.S.: Stability and Nonlinear Solid Mechanics. Wiley \& Son, London (2000)

22. Nagl, M.M., Saunders, S.R.J., Evans, W.T., Hall, D.J.: The Tensile Failure of Nickel Oxide Scales at Ambient and at Growth Temperature. Corrosion Science 35, 965-977 (1993)

23. Marigo, J.J., Truskinovsky, L.: Initiation and propagation of fracture in the models of Griffith and Barenblatt. Continuum Mechanics and Thermodynamics 16(4), 391-409 (2004)

24. Braides, A.: Approximation of Free-Discontinuity Problems, Lecture Notes in Mathematics, vol. 1694. Springer (1998)

25. Giacomini, A.: Ambrosio-Tortorelli approximation of quasi-static evolution of brittle fractures. Calculus of Variations and Partial Differential Equations 22(2), 129-172 (2005)

26. Pham, K., Amor, H., Marigo, J.J., Maurini, C.: Gradient damage models and their use to approximate brittle fracture. International Journal of Damage Mechanics 20(4, SI), 618-652 (2011)

27. Bourdin, B.: Numerical implementation of the variational formulation of quasi-static brittle fracture. Interfaces and Free Boundaries 9, 411-430 (2007) 